\title{
On the Geometry
}

$$
\text { -or- }
$$

\section{The Transformation Group}

$$
p, \quad q, \quad r, \quad x q, \quad y p, \quad z q, \quad x p-y q, \quad z^{2}
$$

BY

J. E. WILLIAMS. 


\section{CONTENTS.}

$\S 1$.

ART.

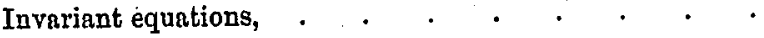

Absolutely invariant points, . . . . . . . . . . . . . . . . . . . . .

Path-ourves;

Path-curres in the $x y$-plane,

Invariants of $n$ points,

$\S 2$.

Invariant Curve framilies.

Differential invariants, Differential parameter,

$\$ 3$.

Equivalence of Curves.

$\S 4$.

Invariant Surface families.

Transformations twice extended, three times extended, Differential parameter for sub-group, 66. ". " complete.system,

$\$ 5$.

Equivalence of Surfaces. 


\section{On the Geometry}

$$
-\mathrm{OF}-
$$

\section{The Transformation Group}

$$
p, \quad q, \quad r, \quad x q, \quad y p, \quad z r, \quad x p-y q, \quad z^{2} r .
$$

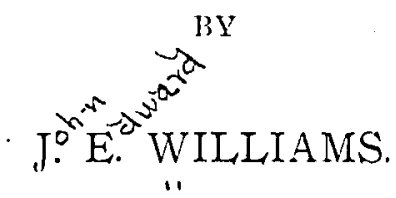

WASHINGTON, D. C. :

Gibson Bros., Printers and Bookbindeks.

1849 . 


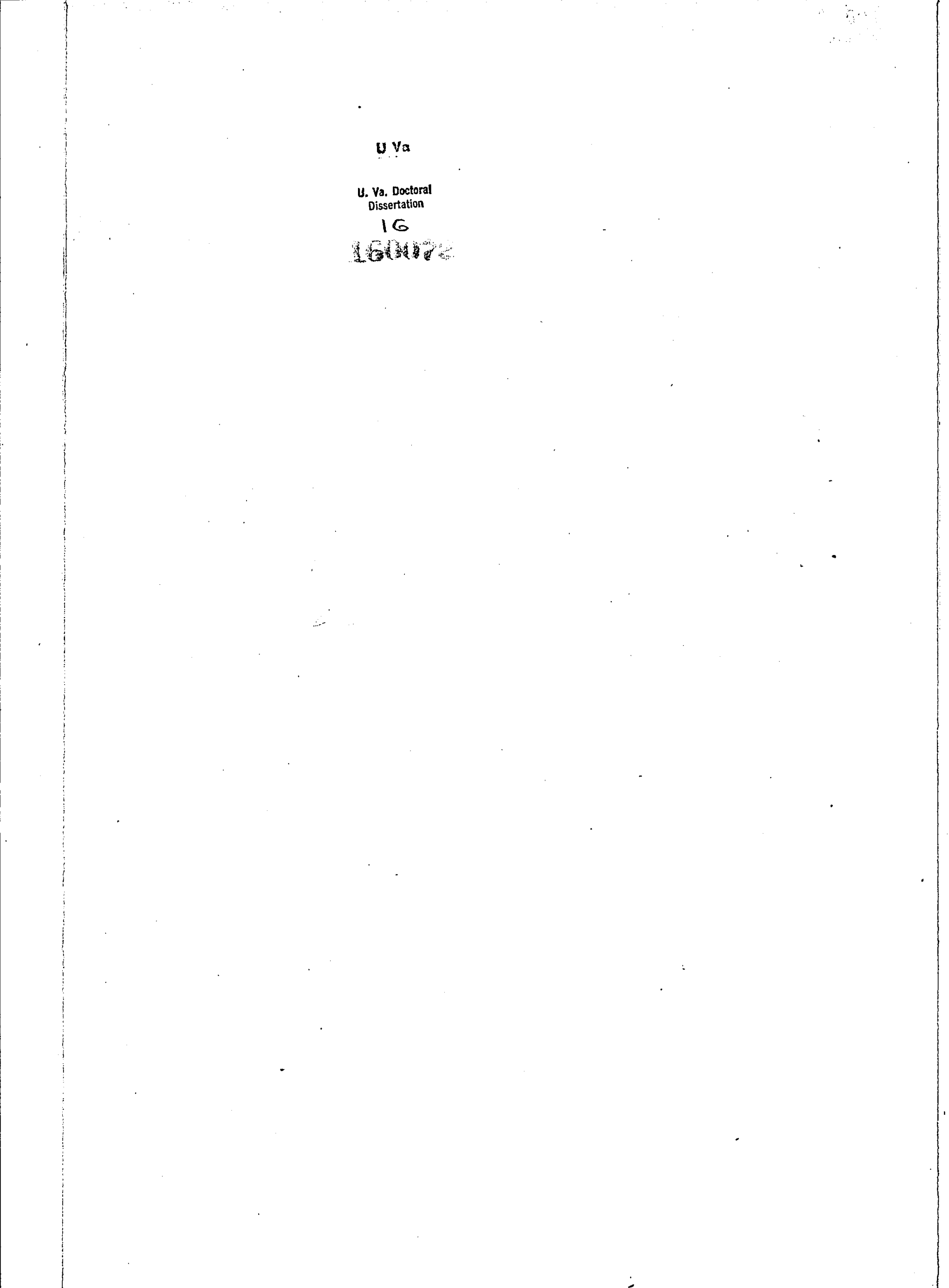




\section{PREFACE.}

Lire.

The author's early training began in the public schools of Charlotte County, Va., principally the High School of Smithville. In the year 1889 he entered Hampden-Sidney College, where he graduated with the A. B. degree at the commencement of 1892. He was then elected principal of the Boydton High Schriol, which position he resigned after two years to become first assistant of the Commerce Street School in Roanoke, Va. After one year's work in this school he returned to Boydton to accept a position as private instructor for Col. Thomas F. Goode. Finally, in the year 1896 he entered the University of Virginia to pursue a special conrse in Pure Mathematics.

The following pages were presented to the Faculty as a dissertation for the degree of Doctor of Philosophy. The subject met with the approval of Dr. J. M. Page, and it may be added that, so far ats conld be learned from the few works on Modern Mithematics in the University library, the investigntious are new.

$$
\text { d. } 19.43
$$

Univinsity of Vinginia, 1899. 


\section{ON THE GEOMETRY OF THE TRANSFORMATION GROUP}

$$
p, \quad q, \quad r, \quad x \eta, \quad y p, \quad z r, \quad x \eta-y \eta, \quad z^{2} r
$$

By J. E. WiLliams.

1. In the following sections we shall not attempt to make an exhaustive discussion of the above group of infinitesimal transformations, but shall limit ourselves to some of the most interesting and important of the investigations which tre possible.

We shall begin by determiuing whether or not any invariant equations exist, and by finding all absolately invauriant points, as well as the path-anrves of the $G_{s}$. We shall limit onr considerations to points, curves, etc., within at finite listance of the origrin.

$$
\text { s. }
$$

2. Representing the transformations of the group by $x_{k,} f$, where $(k=1$ .. 8), we know that if an equation of the form $F(x, y, z)=0$ is invariant under the trausformations of the $G_{s}$, we must have $x_{k}\left(F^{\prime}\right)=0$, either identically or in virtine of $F(x, y, z)=0 . *$

If now we form the matrix of the equations

$$
\begin{aligned}
& X_{1}(F)=\frac{\partial F}{\partial x}+0+0=0 \\
& X_{2}(F)=0+\frac{\partial F}{\partial y}+0=0 \\
& X_{3}(F) \quad 0+0+\frac{\partial F}{\partial z}=0 \\
& X_{4}(F)-0+x \frac{\partial F}{\partial y}+0=0 \\
& X_{5}(F)-y \frac{\partial F}{\partial x}+0+0=0 \\
& X_{6}(F)-0+0+z \frac{\partial F}{\partial z}=0 \\
& X_{5}(F)=x \frac{\partial F}{\partial x}-y \frac{\partial F}{\partial y}+0=0 \\
& X_{3}(F) \equiv 0+0+z^{2} \frac{\partial F}{\partial z}=0
\end{aligned}
$$

* Lie's Coutinuierliche Gruppen, Kap. 16. 
we find for one of the three row determinants

$$
\left|\begin{array}{lll}
1 & 0 & 0 \\
0 & 1 & 0 \\
0 & 0 & 1
\end{array}\right|
$$

which can never be zero. This shows that no systern of values of $x, y, z$ will make all the three row determinants vanish, aud hence we conclude that no equation $F(x, y, z)=0$, that is $n 0$ surface, within a finite distance of the origin, is invariant under every transformation of the $G_{s}$.

3. The most general transformation of the group has the form

$$
\left.X f=(a x+b y+c) p+\left(a_{1}\right)-u y+c_{1}\right) q+\left(a_{2} z^{2}+b_{2} z+c_{2}\right) r=0 .
$$

Since, if a point is absolutely invuriant under If, we must have $i a=i y=$ $\delta z=0$ at that point, all iuvariant points are found from the equations

which give

$$
\begin{gathered}
a x+b y+a=0 \\
a_{1} x-a y+c_{1}=0 \\
a_{2} z+b_{2} z+c_{2}=0 ;
\end{gathered}
$$

$$
x=-\frac{\left(a c+b c_{1}\right)}{a^{2}+a_{1} b}, y=\frac{a c_{1}-a_{1} c}{a^{2}+a_{i} b}, z=-\frac{b_{2} \pm V^{\prime} b_{2}^{2}-4 a_{2} c_{2}}{2} .
$$

These are the coordinates of all points invariant under the general transformation $I f$. By specializing the undetermined constants $a, \ldots, c_{2}$, we find, of course, the point or points iuvariant under each particular transformation of the $G_{b}$. If, in particular, the traustormation reduces to a translation, the point moves off to intinity.

4. We shall now fiud the path-curves of the general trausformation of the $G_{8}$. From equation (1) in Art. 3 we see that they are given by

$$
\frac{d x}{a x+b y+c}=\frac{d y}{a_{1} x-a_{y}+c_{1}}=\frac{d z}{a_{2} z^{2}+b_{2} z+c_{2}} .
$$

The equations in $x, y$ can be made homogeneous by substituting

$$
\bar{x}=x-h, \quad \bar{y}=y-k,
$$

where $h$ and $k$ are determined from the equations

$$
\begin{aligned}
a h+b k+c & =0 \\
a_{1} h-a k+c_{1} & =0 .
\end{aligned}
$$


- wiLlians. ON THE GEONEIRY OF THE 'TRANSFORMation GROUP, ETC.

We then have

$$
\left(a_{1} \bar{x}-a \bar{y}\right) d \bar{x}-(a \bar{x}+b \bar{y}) d \bar{x}=0 .
$$

If now we put in this equation

$$
\bar{y}=z \bar{x}
$$

it has the form

$$
\left(a_{1}-2\left(\iota z-6 z^{2}\right) d \bar{x}-(a+b z) \bar{x} d z=0,\right.
$$

or

$$
\frac{\sqrt{x}}{\bar{x}}+\frac{1}{2} \frac{(-2 a-2 l z) d l z}{a_{1}-2 u z-b z^{2}}=0
$$

Hence

$$
\log \bar{x}+\frac{1}{2} \log \left(a_{1}-2 a z-\lg z^{2}\right)=\frac{1}{2} \log A ;
$$

or substituting

$$
\therefore=\frac{\bar{y}}{\bar{x}}
$$

we easily find

$$
a_{1} \bar{x}^{2}-2 a \bar{x} \bar{y}-b \overline{y^{2}}=A
$$

Lastly, substituting for $\bar{x}$ and $\bar{y}$ their values $x-h$ and $y-k$, respectively, this equation has the form

$$
\begin{array}{r}
a_{1} x^{2}-b y^{2}-2 a x y+\left(2 \iota k-2 \iota_{1} h\right) x+(2 h \iota+2 k b) y+a_{1} h^{2}-2 a h k \\
-8 k^{2}-A=0 .
\end{array}
$$

If now we find $\bar{x}$ in terms of $\bar{y}$, from

$$
a_{1} \bar{x}^{2}-2 \overline{a x y}-b \bar{y}^{2}=A
$$

we have

$$
a_{1} \bar{x}=\bar{u} \bar{y}+1 \overline{y^{2}\left(1+a_{1} b\right)+A a_{1}}
$$

which substituted in

$$
\frac{d \bar{y}}{a_{1} x-a \bar{y}}
$$

gives

$$
\frac{d \bar{y}}{\sqrt{y^{2}\left(1+a_{1} b\right)+A a_{1}}} .
$$


WILLIANS. ON THE GEOMETRY OF THE TIRANSTORMATTON GROUP, ETC.

Hence we get the other integral function of our differential equation from

$$
\frac{\sqrt{y}}{\left(1+a_{1} b\right)^{K_{2}} \sqrt{\bar{y}^{2}+\frac{A a_{1}}{1+u_{1} b}}}-\frac{d z}{a_{2} z^{2}+b_{2} z+c_{2}}=0 .
$$

If $a_{2} c_{2}<\frac{b_{2}^{2}}{4}$, it is easily seen that the integral of this equation is

$\frac{1}{\left(1+a_{i} b\right)^{1 / 2}} \log \left\{\bar{y}+\sqrt{\bar{y}^{2}+\frac{A a_{1}}{1+a_{1} b}}\right\}$

Hence

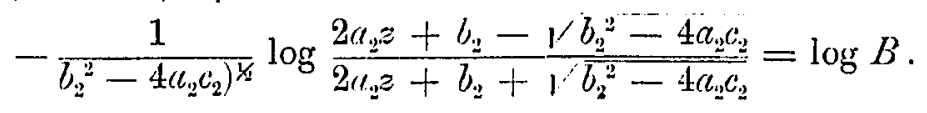

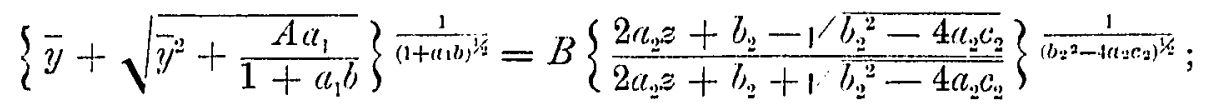

or substituting for $\bar{y}$ and $A$ their values,

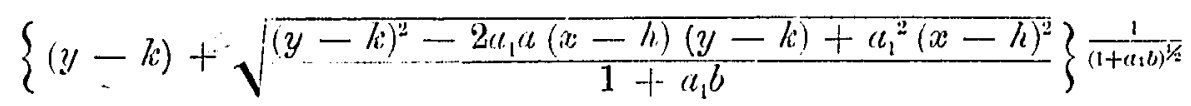

$$
\begin{aligned}
& =B\left\{\frac{2 a_{2} z+b_{2}-1 \overline{b_{2}^{2}-4 a_{2} c_{2}}}{2 a_{2} z+b_{2}+1 \cdot b_{2}^{2}-4 a_{2} c_{2}}\right\} \frac{1}{\left(b_{2}^{2}-4\left(a_{2} c_{2}\right)^{2}\right.} \text {. }
\end{aligned}
$$

We shall not consicler the case when $a_{2} c_{2}>\frac{b_{2}^{2}}{4}$, since in so doing no simple results are obtained.

Equations (2) and (3) taken together represent a family of curves which are called the puth-curves. This family, of course, is invariant in such manner that each curve is absolutely invariant while the points are interchanged among each other.

5. It is interesting to observe that equation (2) represents a family of cylindrical surfaces whose traces in the $x y$-plane are conic sections. If we represent the coordinates of the centre of these conics by $\left(x^{\prime}, y^{\prime}\right)$ and find them in the usual way, it is easily seen that

$$
\begin{aligned}
& x^{\prime}=-\frac{a(h a+h b)+b\left(-h a_{1}+k a\right)}{-\left(a_{1} b+a^{2}\right)}=h, \\
& y^{\prime}=-\frac{a\left(-h a_{1}+k a\right)-a_{1}(h a+k b)}{-\left(a_{1} b+a^{2}\right)}=k,
\end{aligned}
$$

where, as we have seen, $h$ and $k$ are found from the equations

$$
\begin{aligned}
& a h+b k+c=0, \\
& a_{1} h-a k+c_{1}=0 .
\end{aligned}
$$


WILLIAMS. ON THE GEOMETRY OF THE TRANSFORMATION GROUP, ETC.

Hence

$$
a^{\prime}=h=-\frac{a c+b c_{1}}{a^{2}+a_{1} b} ; \quad y^{\prime}=h=\frac{a c_{1}-a_{1} c}{a^{2}+a_{1} b}
$$

Now this is the point which we found to be absolutely invariant under $X f$, in the : $x y$-pliuse. That is, the conics which we foumd as the path-curves in the $x y$-plane have for their centre the point which is absolutely invariant.

A number of interesting problems suggest themselves in connection with this part of the subject. However we shall conchde this section with a remark on the invariants* of $n$ points under the transformations of the $G_{*}$.

6. If $"$ points hive an invirint nucler the $G_{s}^{\prime}$, it is clear that for some function $f^{\prime}\left(x_{l}, y_{i}, z_{i}\right)$ we must lave for ench transformation of the $G_{s}$ :

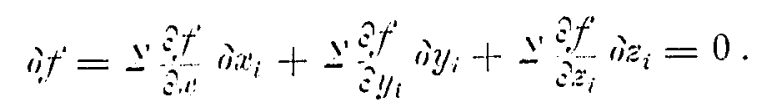

It is also clear that an invariant of the transformations containing $x$ and $y$ is, at the sime time, invariant under those contining a only, aud vice verst. We have then from (1); omitting for the present the equations containing $z$,

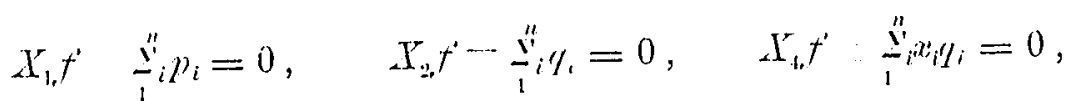

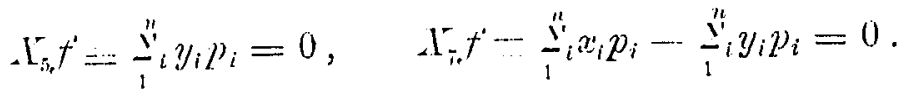

'This is a complete system of tive members in $2 n$ variables, so that there wre $2 n-5$ solutions. The solutions common to $X_{1} t=0$ and $X_{2 .} t^{\prime}=0$ are seen to be

$$
x_{1}-x_{i}-u_{i} \text { and } y_{1}-y_{i}-u_{j}, \text { where } i=2 \ldots n, j=i+n-2
$$

If now these solutions be introluced into the other equations, the latter hecome

$$
\begin{aligned}
& X_{1} f^{\prime} \equiv \Sigma^{\prime} u_{i} \frac{\partial f^{\prime}}{\partial u_{j}}=0 \\
& X_{i_{0}} f^{\prime}=\Sigma^{\prime} u_{j} \frac{\partial f^{\prime}}{\partial u_{i}}=0 \\
& X_{i} f=\Sigma^{\prime} u_{i} \frac{\partial f}{\partial u_{i}}-\Sigma^{\prime} u_{j} \frac{\partial f}{\partial u_{j}}=0, i=1, \ldots, n-1, j=i,+n-1 .
\end{aligned}
$$

The solutious of $X_{i} f^{\prime}=0$ are

$$
\frac{u_{1}}{u_{i}}, u_{1} u_{j}, \quad \text { where } i=2 \ldots n-1, j=n \ldots 2 n-3 .
$$


Writing $\frac{u_{1}}{u_{2}} \ldots u_{1} u_{2 n-3}=v_{1} \ldots v_{2 n-3}$, the two remaining equations become expressed in terms of the $v_{i}$

$$
\begin{aligned}
& \bar{X}_{i} f=2 \frac{1}{v_{i}} \frac{\partial f}{\partial v_{j}}, \quad i=0 \ldots n-2 ; j=i+n-1, v_{0}=1 \\
& X_{i} f=\Sigma^{\prime}\left(v_{i} v_{n-1}-v_{i}^{2} v_{j}\right) \frac{\partial f}{\partial v_{i}}+\Sigma^{\prime}\left(v_{n-1} v_{s}\right) \frac{\partial f}{\partial v_{s}}=0, \\
& i=1 \ldots n-2, j=i+n-1, s=n-1 \ldots 2 n-3 \text {. }
\end{aligned}
$$

The solutions of $I_{\mathrm{l}} f=0$ are

$$
v_{i}, v_{n-1}-v_{i} v_{j+n-1}, \quad \text { where } i=1 \ldots n-2 .
$$

Finally writing for $v_{1} \ldots v_{n-1}-\dot{v}_{n-1} v_{2 n-3}$ respectively $v_{1} \ldots v_{2 n-3,3}$ and introducing these solutions into $X_{1} f=0$, we have

$$
X_{4} f=\frac{y}{\prime} w_{i} w_{j} \frac{\partial f}{\partial w_{i}}+\Sigma^{\prime} v_{j}^{2} \frac{\partial f}{\partial v_{j}}=0, \quad i=1 \ldots n-2, j=i+n-2 .
$$

Hence we find that $n-2$ of the common solutions have the forms

while $n-3$ have the forms

$$
J_{i}=\frac{w_{i+n-1}}{w_{i}}, \quad i=1 \ldots n-2
$$

$$
Q_{s}=\frac{w_{n-1}-w_{s}}{w_{n-1} w_{s}}, \quad s=n \ldots, 2 n-4 .
$$

The $A_{i}$ considered in the plane are nothing but the double areas of triangles; and it is ensily seen that we can form $n-3$ other independent functions of the $J_{i}$ and $Q_{s}$ which will also be donble areas of triangles.

Considered in space, since the abore solutions satisfy the equations in $z$ also, these results show that the projections of all areas on the $x y$-plane remain invariant under all transformations of the $G_{\mathrm{s}}$.

As we have said, the solutions obtained from the equations in $z$ will also be invariants of the whole group. Here, as we shall see, it is only mecessary to consider the case with four variables, whence general results may be obtained by inspection. We have then

$$
\begin{aligned}
& X_{b_{4}} f=r_{1}+r_{2}+r_{3}+r_{4}=0 \\
& X_{7} f-z_{1} r_{1}+z_{2} r_{2}+z_{3} r_{3}+z_{4} r_{4}=0 \\
& X_{\delta_{1}} f=z_{1}{ }^{2} r_{1}+z_{2}{ }^{2} r_{2}+z_{3}{ }^{2} r_{3}+z_{4}{ }^{2} r_{4}=0 .
\end{aligned}
$$


WILLIAMS. ON THE GEONETIRY OF THE TRANSFORMATION GROUP, ETC.

The solutions common to $X_{0} f^{\prime}=0$ and $X_{7} f^{\prime}=0$ are easily found to be

$$
\frac{z_{1}-z_{2}}{z_{2}-z_{3}}=v_{1}, \quad \frac{z_{3}-z_{4}}{z_{2}-z_{3}}=v_{2} .
$$

The remaining equation, when these variables are introduced, takes the form

$$
v_{1}\left(v_{1}+1\right) \frac{\partial f}{\partial v_{1}}-v_{2}\left(v_{2}+1\right) \frac{\partial f}{\partial v_{2}}=0
$$

The solution to this equation is

$$
\frac{v_{1}}{v_{1}+1} \cdot \frac{v_{2}}{v_{2}+1}=\left[\frac{z_{1}-z_{2}}{z_{1}-z_{3}}\right]\left[\begin{array}{l}
z_{3}-z_{4} \\
z_{2}-z_{4}
\end{array}\right]
$$

which is the anharmonic ratio of the $z$ ordinates of the four points.

It is clear that if we consider $n$ points, the results will be $n-3$ independent anharmonic ratios of the $n$ points taken four at a time. This is equivalent to saying that the anbarmonic ratio of any four planes parallel to the $x y$-plane is invariant under the $G_{\mathrm{s}}$. Thus we find that $n$ points have $3 n-8$ invariants under the $G_{s}$, of which $n-3$ contain only the variables $z_{i}$, while the others contain only the $x_{i}$ and $y_{i}$.

If now we consider only two points, the complete system consists of eight members in only six variables, and therefore has no solution. We can, however, form the matrix of the equations and determine whether or not any relations exist between the elements such as will make all the six row determinants vanish. Such relations, if any exist, may be seen to be invariant. The matrix is

$$
\left|\begin{array}{cccccc}
1 & 1 & 0 & 0 & 0 & 0 \\
0 & 0 & 1 & 1 & 0 & 0 \\
0 & 0 & x_{1} & x_{2} & 0 & 0 \\
y_{1} & y_{:} & 0 & 0 & 0 & 0 \\
x_{1} & x_{2} & -y_{1} & -y_{2} & 0 & 0 \\
0 & 0 & 0 & 0 & 1 & 1 \\
0 & 0 & 0 & 0 & z_{1} & z_{2} \\
0 & 0 & 0 & 0 & z_{1}^{2} & z_{2}^{2}
\end{array}\right| .
$$

It is readily seen that if $x_{1}=x_{2}$ and $y_{1}=y_{2}$ at the same time, all the six row determinants of this matrix will vinish, siuce in ench two columns will be 
identical. Also if $z_{1}=z_{2}$ each determinant will vanish for the same reason. We therefore have two invariant relations, namely, $z_{1}=z_{3}$, and the simultaneous relations $x_{1}=x_{2}$ and $y_{1}=y_{2}$.

Now the transformation

$$
(a x+b y+c) p+\left(a_{1} x-a y+a_{1}\right) q
$$

in the xy-plane leaves the point given by

$$
\left.\begin{array}{c}
a x+b y+c=0 \\
a_{1} x-u y+a_{1}=0
\end{array}\right\}
$$

invariant. Let this point be $x^{\prime}, y^{\prime}$. If another point $x_{2}, y_{2}$ is held, both $a^{\prime}, y^{\prime}$ and $a_{.}, y_{2}$ must satisfy (1). But this is impossible unless $a^{2}+a_{1} b=0$; that is, the equations wonld not be inclepenclent. Hence if we hold $x_{2}, y_{2}$ all points in the $x y$-pline are absolutely invariant. Hence the invariant simultaneons relations $x_{1}=x_{2}$ and $y_{1}=y_{2}$ men that if we hold $a_{2}, y_{2}$, the point $a, y, z$ and only move aloug the line

$$
\left.\begin{array}{l}
x_{1}=x_{2} \\
y_{1}=y_{2}
\end{array}\right\} .
$$

The invariant relation $z_{1}=z_{2}$ means that if the points lie in a pline before they are trausformed, they lie in a plawe after they are transformed.

By a somewhat similar process, we could find the invariants of $m$ poiuts and $n$ planes.

\section{$\$ 2$.}

7. We will now extend the transformations of the group with a view to finding the differential invariant of the lowest order, and subsequently all those of higher orders, where $y$ and $z$ are functions of $x$. We will in general write

From

$$
\frac{d^{\prime \prime} y}{d x^{\prime \prime}} \quad y_{11}, \frac{d^{\prime \prime} \cdot z}{d x^{\prime \prime}}-z_{n}
$$

$$
d y_{n-1}-y_{n} d x \quad 0
$$

we have at once the increment which $y_{n}$ receives by means of any infinitesimal transformation in the form

$$
i y_{n}=\frac{d \partial y_{11-1}}{d x}-y_{n} \frac{d \partial x}{d x}
$$


and similarly for $\approx_{n}$

$$
d z_{n}=\frac{d d z_{n-1}}{d x}-z_{n} \frac{d \partial x}{d x}
$$

The trinsformations, as is well known, are extended by means of these formulie. In order to find the differential invariant of the lowest order, we inust extend the transformations three times and equate the results to zero, since that will give a complete system of eight members in nine variables, and therefore one solution. If we extend less than three times, it may be seen that we have more independent equations than variables, so that according to the general theory of the complete system no solution exists. Hence the clifferential invariant of the lowest order will be of the third order. Thus extending by means of (1) and (2), we have

$$
\begin{aligned}
& X_{1 .} f=\frac{\partial f}{\partial x}=0 \\
& X_{i t} t^{\prime}=\frac{\partial f^{\prime}}{\partial y}=0 \\
& X_{3,} t^{\prime}=\hat{c} f^{\prime}=0 \\
& X_{1} f^{\prime}=x \frac{\hat{\partial} f^{\prime}}{\hat{\partial} y}+\frac{\partial f}{\partial y_{1}}=0
\end{aligned}
$$

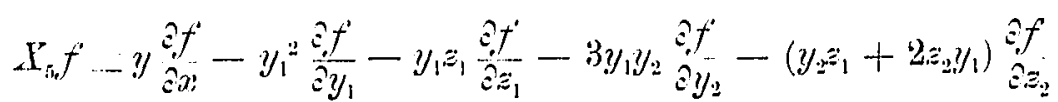

$$
\begin{aligned}
& -\left(4 y_{1} y_{3}+3 y_{2}{ }^{2}\right) \frac{\partial f^{\prime}}{\partial y_{3}}-\left(y_{3} z_{1}+3 y_{2} z_{2}+3 y_{1} z_{3}\right) \frac{\partial f^{\prime}}{\partial z_{3}}=0
\end{aligned}
$$

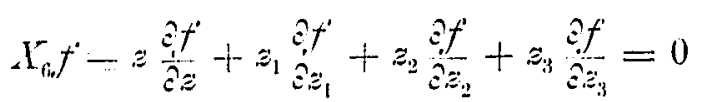

$$
\begin{aligned}
& X_{i} f-a \frac{\partial f}{\partial x}-y^{\prime} \partial f-2 y_{1} \frac{\partial f}{\partial y_{1}}-z_{1} \frac{\partial f}{\partial z_{1}}-3 y_{2} \frac{\partial f}{\partial y_{2}}-2 z_{2} \frac{\partial f}{\partial z_{2}}-4 y_{3} \frac{\partial f}{\partial y_{3}} \\
& -3 \varepsilon_{3} \frac{a^{\prime}}{a z_{3}}=0
\end{aligned}
$$

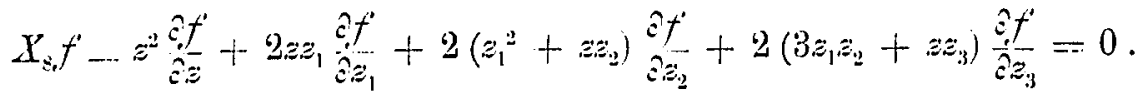

The tirst fonr equations show that $x, y, z, y_{1}$ are not contained in the solution sought. The cumplete system can thus be written in the form 
14 WILLIAMS. ON THE GEONETRY OF 'THE TRANSFORMATION GROUP, WTC.

$$
\begin{aligned}
& X_{i_{1}} f=y_{1} z_{1} \frac{\partial f}{\partial z_{1}}+3 y_{1} y_{2} \frac{\partial f}{\partial y_{2}}+\left(y_{2} z_{1}+2 y_{1} z_{2}\right) \frac{\partial f}{\partial z_{2}}+\left(4 y_{1} y_{3}+3 y_{2}^{2}\right) \frac{\partial f}{\partial y_{3}} \\
& +\left(y_{3} z_{1}+3 y_{2} z_{2}+3 y_{1} z_{3}\right) \frac{\partial f}{\partial z_{3}}=0 \\
& I_{i 2} f \quad z_{1} \frac{\partial f}{\partial z_{1}}+z_{2} \frac{\partial f}{\partial z_{2}}+z_{3} \frac{\partial f}{z_{3} z_{3}}=0 \\
& x_{i} f=z_{1} \frac{\partial f}{\partial z_{1}}+3 y_{2} \frac{\partial f}{\partial y_{2}}+2 z_{2} \frac{\partial f}{\partial z_{2}}+4 y_{3} \frac{\partial f}{\partial y_{3}}+3 z_{3} \frac{\partial f}{\partial z_{3}}=0 \\
& X_{z_{1}} f=z z_{1} \frac{\partial f}{\partial z_{1}}+\left(z_{1}{ }^{2}+z z_{2}\right) \frac{\partial f}{\partial z_{2}}+\left(3 z_{1} z_{2}+z z_{3}\right) \frac{\partial f}{\partial z_{3}}=0 \text {. }
\end{aligned}
$$

These equations can be greatly simplified by algebraic reduction. Replace

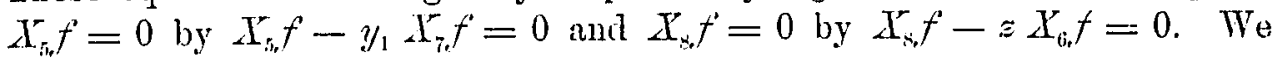
then liave

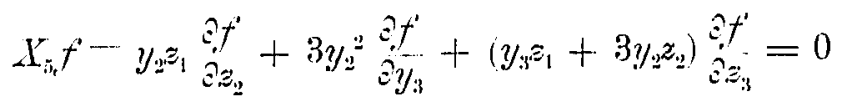

$$
\begin{aligned}
& y_{8} f=z_{1} \frac{\partial f}{\partial z_{1}}+z_{2} \frac{\partial f}{\partial z_{2}}+z_{3} \frac{\partial f}{\partial z_{3}}=0 \\
& I_{i} f \cdots z_{1} \frac{\partial f}{\partial z_{1}}+3 y \frac{\partial f}{\partial y_{2}}+2 z_{2} \frac{\partial f}{\partial z_{2}}+4 y_{3} \frac{\partial f}{\partial y_{3}}+3 z_{3} \frac{\partial f}{\partial z_{3}}=0 \\
& r_{x_{0}} f^{2} \quad z_{1} \frac{\partial f}{\partial z_{2}}+3 z_{2} \frac{\partial f}{\partial z_{3}}=0 \text {. }
\end{aligned}
$$

Again, replice in these equations $X_{i_{n}} f^{\prime}=0$ by $X_{i_{r}} f^{\prime}-y_{2} J_{s_{1}} f^{\prime}=0$ and $X_{i_{0}} f=0$ by $I_{i,} f^{\prime}-X_{i,} f=0$, and the equations are reduced to the forms

$$
\begin{aligned}
& I_{i_{r}} f \quad 3 y_{2}^{2} \frac{\partial f}{\partial y_{3}}+y_{3} z_{1} \frac{\partial f}{\partial z_{3}}=0 \\
& I_{b_{r}} f^{\prime}=z_{1} \frac{\partial f}{\partial z_{1}}+z_{2} \frac{\partial f}{\partial z_{2}}+z_{3} \frac{\partial f}{\partial z_{3}}=0 \\
& X_{r_{r}} f \quad 3 y_{2} \frac{\partial f}{\partial y_{2}}+z_{2} \frac{\partial f}{\partial z_{2}}+4 y_{3} \frac{\partial f^{\prime}}{\partial y_{3}}+2 z_{3} \frac{\partial f}{\partial z_{3}}=0 \\
& I_{z_{2}} f^{\prime}-z_{1} \frac{\partial f}{\partial z_{2}}+3 z_{2} \frac{\partial f}{\partial z_{3}}=0 .
\end{aligned}
$$

It now remains to find the solution to this complete system. As an explatnation of the method by which we shitl proceed, we recall the following theorem from the theory of the complete system.

$$
\text { If } A_{1} f^{\prime}=0 \ldots A_{r_{2}} f^{\prime}=0 \text {, form a complete systom in the variables } x_{1} \ldots x_{m}
$$


WILLIAMS. ON THE GEOMETRY OF THE TRANSFONMATION GROUP, ETC. · 15 the integration of the same can be accomplished in the following manner. We seek the solutions $\varphi_{1} \ldots \varphi_{n-1}$ of $A_{1} f=0$; then, form

$$
\Lambda_{2} f=\Lambda_{2} \varphi_{1} \frac{\partial f}{\partial \varphi_{1}}+\ldots+A_{2} \varphi_{n-1} \frac{\partial f}{\partial \varphi_{n-1}}=0 \text {. }
$$

If the ratios of the $A_{2} \varphi_{k}$ are not functions of $\varphi_{1} \ldots \varphi_{n-1}$ alone, the equation $A_{2_{1}} f=0$ will always breck up into several enuations. We integrate one of these cond introduce its solutions $d_{1}^{\prime} \ldots \psi_{n-2}^{\prime}$ into $A_{3,} f=0$. The resulting equertion

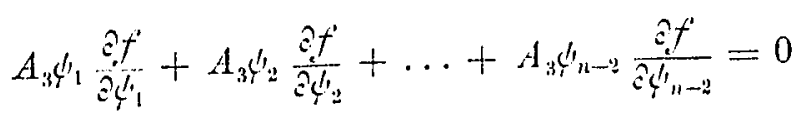

is treated in "similer manner, and so on. If $r<n$ we find ultimately the $n-r$ solutions of the complete sysklem.

Now the solutions of $X_{5} f^{\prime}=0$ are

$$
z_{1}, y_{2}, z_{2}, \text { and } y_{3}{ }^{2} z_{1}-6 y_{2} z_{3}-16
$$

Introducing these solutions as above inclicated, the remaining equations assume the forms

$$
\begin{aligned}
& I_{i_{2}} f \cdots z_{1} \frac{\partial t}{\partial z_{1}}+z_{2} \frac{\partial f}{\partial z_{2}}+u \frac{\partial t}{\hat{\partial} u}=0 \\
& X_{i} t^{\prime} \quad 3 y_{2} \frac{\partial f^{\prime}}{\partial y_{2}}+z_{2} \frac{\partial f^{\prime}}{\partial z_{2}}+s_{n \prime \prime} \frac{\partial f^{\prime}}{\partial \| \prime}=0 \\
& X_{x_{2}} f^{\prime}-z_{1} \frac{\partial f}{\partial z_{2}}-18 y_{2} z_{2} \frac{\partial f}{\partial u}=0 \text {. }
\end{aligned}
$$

The solntions to $X_{t i,} f=0$ are easily found to bes

$$
y=\frac{z_{2}}{z_{1}}-v, \stackrel{z}{z_{1}}-w
$$

Iutrodnce these into the other two equations and they have the forms

$$
\begin{aligned}
& X_{i} f=3 y_{2} \frac{\partial f}{\partial y_{2}}+v \frac{\partial f}{\partial t}+8 w \frac{\partial f}{\partial t}=0 \\
& X_{8} f^{\prime}-\frac{\partial f}{\partial v}-18 y_{2}{ }^{2} v \frac{\partial f}{\partial v}=0 .
\end{aligned}
$$

Finally the solutions of $X_{*} f^{\prime}=0$ are

and $X_{7} f$ becomes

$$
y_{2} \text { and } 9 y_{2} v^{2}+w-v_{1}
$$

$$
X_{i} f-3 y_{2} \frac{\partial f}{\partial y_{2}}+8 v_{1} \frac{\partial f}{\partial v_{1}}=0 .
$$


16 WILLians. ON 'THE GEOMETRY OF THE tRanSFormation Group, ETC.

Hence the common solution of all the equations, that is the solution of the complete system, is found to be

$$
I_{3}: \frac{v_{1}}{y_{2}^{8 / 3}}=\frac{9 y_{2}^{2} z_{2}^{2}+z_{1}\left(z_{1} y_{3}^{2}-6 y_{2}^{2} z_{3}\right)}{z_{1}^{2} y_{2}^{8 / 3}}
$$

This then is the differential invariant of the lowest order. Those of higher orders can be found by further extending the transformations, and for future use we shall find two of the fourth order. Extending as above, and omitting terms containing $i, y, z, y_{1}$, since these variables do not occur in the solutions, we have

$$
\begin{aligned}
& X_{n} f-y_{1} z_{1} \frac{\partial f}{\partial z_{1}}+3 y_{1} y_{2} \frac{\partial f}{\partial y_{2}}+\left(y_{2} z_{1}+2 y_{1} z_{2}\right) \frac{\partial f}{\partial z_{2}}+\left(4 y_{1} y_{3}+3 y_{2}\right) \frac{\partial f}{\partial y_{3}} \\
& +\left(y_{: z_{1}}+3 y_{2} z_{2}+3 y_{1} z_{3}\right) \frac{\partial f}{\partial z_{3}}+\left(5 y_{1} y_{4}+10 y_{2} y_{3}\right) \frac{\partial f}{\partial y_{1}}=0 \\
& X_{6} f=z_{1} \frac{\partial f}{\partial z_{1}}+z_{2} \frac{\partial f}{\partial z_{2}}+z_{3} \frac{\partial f}{\partial z_{3}}=0 \\
& X_{7} f-z_{1} \frac{\partial f}{\partial z_{1}}+3 y_{2} \frac{\partial f}{\partial y_{2}}+2 z_{2} \frac{\partial f}{\partial z_{2}}+4 y_{3} \frac{\partial f}{\partial y_{3}}+3 z_{3} \frac{\partial f}{\partial z_{3}}+5 y_{4} \frac{\partial f^{\prime}}{\partial y_{1}}=0 \\
& \chi_{\mathrm{H}_{2}} f=z_{1} \frac{\partial f}{\partial z_{1}}+\left(z_{1}{ }^{2}+z z_{2}\right) \frac{\partial f}{\partial z_{2}}+\left(3 z_{1} z_{2}+z z_{3}\right) \frac{\partial f}{\partial z_{3}}=0 .
\end{aligned}
$$

These equations can be simplified exictly as in the preceding case, and we have after this reduction

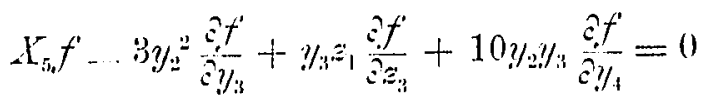

$$
\begin{aligned}
& I_{t i} f \quad z_{1} \frac{\partial f}{\partial z_{1}}+z_{i} \frac{\partial f}{\partial z_{2}}+z_{3} \frac{\partial f}{\partial z_{3}}=0
\end{aligned}
$$

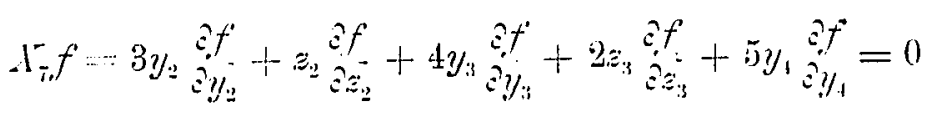

$$
\begin{aligned}
& X_{f} f^{\prime}=z_{1} \frac{\partial f^{\prime}}{\partial z_{2}}+3 z_{2} \frac{\partial f^{\prime}}{\partial z_{3}}=0
\end{aligned}
$$

The solutions of $X_{s}^{\prime} f^{\prime}=0$ are

$$
z_{1}, y / 4, y / 3, y, \text { and } 3 z_{2}^{2}-2 z_{1} z_{3}=\ldots 11
$$


WILLIAMS. ON THE GEOMETRY OF 'IHE TRATSFORMATION GROUP, ITC.

These solutions introduced into the other equations give

$$
\begin{aligned}
& x_{5 i} f=3 y_{2}^{2} \frac{\partial f}{\partial y_{3}}+10 y_{2} y_{3} \frac{\partial f}{\partial y_{3}}-2 y_{3} z_{1}^{2} \frac{\partial f}{\partial u}=0 \\
& X_{i t} f^{\prime}=z_{1} \frac{\partial f}{\partial z_{1}}+2 u \frac{\partial f}{\partial u}=0 \\
& x_{7} f^{\prime}: 3 y_{2} \frac{\partial f}{\partial y_{2}}+4 y_{3} \frac{\partial f}{\partial y_{3}}+5 y_{4} \frac{\partial f}{\partial y_{4}}+2 u \frac{\partial f}{\partial u}=0 .
\end{aligned}
$$

If we next take $X_{\text {ir }} f^{\prime}=0$ we have as solutions

$$
y_{2}, y_{3}, y_{4}, \frac{u}{z_{1}^{2}}=. . v
$$

and in these variables the other two equations have the forms

$$
\begin{aligned}
& X_{5} f \quad 3 y_{2}^{2} \frac{\partial f}{\partial y / 3}+10 y_{2} y_{3} \frac{\partial f}{\partial y_{4}}-2 y_{3} \frac{\partial f}{\partial v}=0 \\
& x_{7} f^{\prime}=3 y_{2} \frac{\partial f}{\partial y_{2}}+4 y_{3} \frac{\partial f}{\partial y / 3}+\bar{v} y_{1} \frac{\partial f}{\partial y}+2 v \frac{\partial f}{\partial v}=0 .
\end{aligned}
$$

We next find the solntions of $\lambda_{n,} f^{\prime}=0$ to be

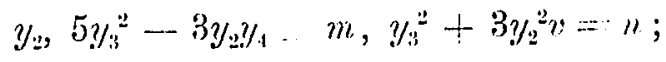

and $X_{i}^{-} f$ assumes the form

Hence we find

$$
r_{i} f \quad 3 y \frac{\partial f}{\partial y_{2}}+8 m \frac{\partial f}{\partial m}+8 n \frac{\partial f}{\partial n}=0 .
$$

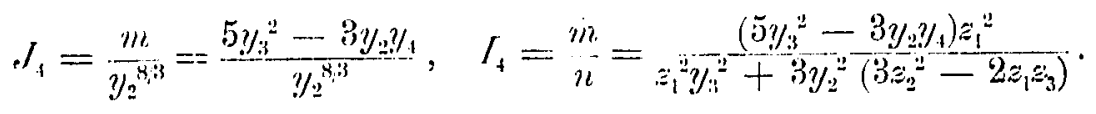

This process of extending the transformations conld be kept up indefivitely, and the differential invariants of any clesired order conld be found, theoretically; but such a process would soon become very cumbersome, so that we shall in the next anticle give a more conrenient method by which all the differential invariants of an order higher than the fourth can be found by mere differentiation.

S. Tuet $\varphi$ he a differential invariant of the lowest orler of the $t_{4}$; we now

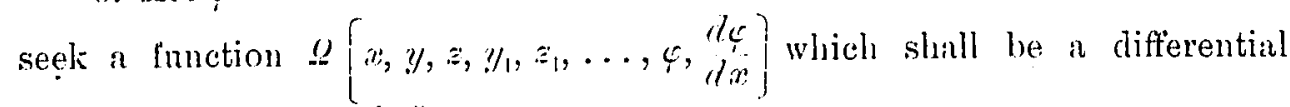
invariant whenever ç is.: 
WILLIAMS. ON THE GEOMETRY OF 'IHE 'IRANSFOMNATION GROUP, EYC.

Indicating by is the increment received by means of an infinitesimal trausformation of the group, since

we have

$$
d_{c}=\varphi^{\prime} d x
$$

$$
\operatorname{sil} \varphi \cdot \cdots \varphi^{\prime} \cdot d x+\varphi^{\prime} \cdot \partial d x
$$

or since $d$ and $i$ can be interchanged,

$$
\Delta \varphi^{\prime}-\frac{d i c}{d x}-\varphi^{\prime} d x
$$

Now since $\varphi$ is to be invariant, it receives no increment; that is $i c=0$. Hence

$$
r \varphi^{\prime}=-\varphi^{\prime} \frac{d \partial x}{d x}
$$

If in this equation we substitute for $\lambda_{2}$ its values as given by the trausformations $y, q, r, x q$, we fiud in each case that $i \varphi^{\prime}=0$. For $y p$, we lave $i x=\eta$ and therefore $i \varphi^{\prime}=-\varphi^{\prime} y_{1}$. In the same way we may find the increments which $\varphi^{\prime}$ receives by means of the other transformations.

Extending now the transformations so as to have a complete system of eight members in nine variables, and omitting terms (Art. 7) containing $\frac{\partial Q}{\partial x}, \frac{\partial Q}{\partial y}, \frac{\partial Q}{\partial z}, \frac{\partial Q}{\partial y_{1}}$, it is easily seen that one complete system has the form

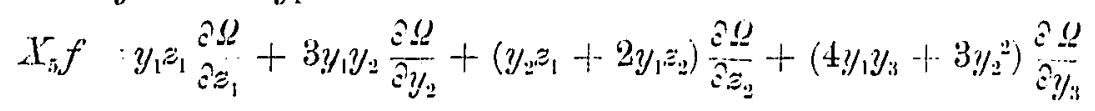

$X_{b_{n}} f-z_{1} \frac{\partial !}{\partial z_{1}}+\hat{z}_{2} \frac{\underline{\partial}}{\partial z_{2}}=0$ $+\varphi^{\prime} y_{1} \frac{\hat{c} !}{\hat{i} \varphi^{\prime}}=0$

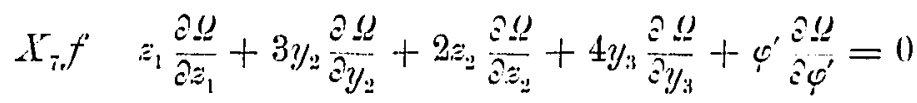

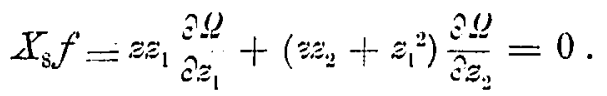

In these equations replace $X_{s_{1}} f=0$ by $X_{i_{1}} f^{\prime}-y_{1} X_{i_{1}} f^{\prime}=0$, aud $X_{s_{2}} f^{\prime}=0$ by $X_{\varepsilon} f-z X_{6} f=0$. We then have

$$
\begin{aligned}
& X_{i_{1}} f-y_{z_{2} z_{1}} \frac{\partial Q}{\partial z_{2}}+3 y_{2}^{2} \frac{\partial Q}{\partial y_{3}}=0 \\
& X_{0} f \equiv z_{1} \frac{\partial Q}{\partial z_{1}}+z_{2} \frac{\partial Q}{\partial z_{2}}=0 \\
& X_{i} f^{\prime}-z_{1} \frac{\partial Q}{\partial z_{1}}+3 y_{2} \frac{\partial Q}{\partial y_{2}}+2 z_{2} \frac{\partial Q}{\partial z_{2}}+4 y_{3} \frac{\partial U}{\partial y_{3}}+\varphi^{\prime} \frac{\partial Q}{\partial \varphi^{\prime}}=0 \\
& X_{s} f=z_{1}{ }^{2} \frac{\partial Q}{\partial z_{2}}=0 .
\end{aligned}
$$


WILLIAMS. ON 'THE GEOME'IRI OF 'THE 'TRANSFORMA'TION GROUP, ETC.

The last equation shows that $z_{2}$ is not contained in the solution; and since this is the case, the first and second show that the solution is also free of $\approx_{1}$ and $y_{3 .}$. Hence, we find the common solution from

which we write in the form

$$
\frac{d y_{2}}{3 y_{2}}=\frac{d \varphi^{\prime}}{\varphi^{\prime}}
$$

$$
J \varphi=\frac{\varphi^{\prime}}{y_{2}{ }^{1 / 3}}
$$

Hence, whenever $\varphi$ is a differential invariant, $J_{\varphi}$ is a differential invariant of the next higher order. Therefore, by means of $d \varphi$, which is called the differential paraneter, we can find from any differential invariant one of a higher order by simply differentiating the given differential invariant totally with respect to $x$ and multiplying the result by $1 / y_{2}^{1 / 3}$. Thus

$$
J\left(J_{4}\right)=\frac{45 y_{2} y_{3} y_{4}-9 y_{2}^{2} y_{5}-40 y_{3}^{3}}{3 y_{3}^{4}} \quad J_{5}
$$

is a differential invariaint of the fifth order. Also

$$
J\left(I_{4}\right)=-\Gamma_{5}
$$

is a differential invariant of the fifth order, which is clearly independent of $J_{5}$.

In the same manner we see that

$$
\left.\left.J(\lrcorner\left(J_{4}\right)\right), \quad J^{\prime}\left(J_{4}\right)=\right\lrcorner\left(J_{i}\right)
$$

is a differential invariant of the sixth order; and universally

$$
J^{\prime \prime}\left(J_{4}\right) \text { and } J^{\prime \prime}\left(I_{4}\right)
$$

are two independent differential invariants of the $(n+4)$ th order. Thus it is clear that we can write 'lown all the differential invariants of any required order.

We have reserved the finding of the invariant differential equations and in variant systems from the determinants of the equations forming the complete systems for the next section, wherein we shall also discuss the equivalence of curves.

\section{$\$ 3$.}

Equivulence of Curves.

9. If we extend the transformations of our $G_{s}$ once, it is easily seen from the determinants of the matrix so formed that the only invariant differential equation of the $I .0$ is $z_{1}=0$. 
If we extend twice, we have the matrix

$$
\left\|\begin{array}{ccccccc}
1 & 0 & 0 & 0 & 0 & 0 & 0 \\
0 & 1 & 0 & 0 & 0 & 0 & 0 \\
0 & 0 & 1 & 0 & 0 & 0 & 0 \\
0 & x & 0 & 1 & 0 & 0 & 0 \\
y & 0 & 0 & -y_{1}^{2} & -y_{1} z_{1} & -3 y_{1} y_{2} & -\left(y_{2} z_{1}+2 y_{1} z_{2}\right) \\
0 & 0 & z & 0 & z_{1} & 0 & z_{2} \\
x & -y & 0 & -2 y_{1} & -z_{1} & -3 y_{2} & -2 z_{2} \\
0 & 0 & z^{2} & 0 & 2 z_{1} & 0 & 2\left(z_{1}^{2}+z z_{2}\right)
\end{array}\right\| .
$$

Inclicating by $d_{t}$ the determinant formed from this matrix by suppressing the ith row, we easily find

$$
\begin{aligned}
& \left.J_{s}=3 y_{2}^{2} z_{1}{ }^{2}, \quad\right\lrcorner_{1}-2 z_{1}^{3} y_{1} y_{2}, \quad J_{1}:-6 y_{2}^{2} z_{1} z_{1}{ }^{2}, \quad J_{3}=-6 y_{2} z_{1}^{3}, \quad J_{4}-6 y_{1}^{2} y_{2} z_{1}^{3}, \\
& J_{3}=-3 y_{2}^{2} z_{1}^{3} z^{2}, \quad J_{2}=6 y_{1} y_{2} z_{1}\left(x y_{1}-y\right), \quad J_{1}=6 y_{2} z_{1}^{3}\left(y-x y_{1}\right) .
\end{aligned}
$$

Since $z_{1}$ is the only factor common to all these determinants, the only invariant differential equation is $z_{1}=0$. If, however, we write $I_{3}=\infty$, we find that $y_{2}=0$ is an invariant equation of $I J .0$. We also see that $z_{1}=y_{2}=0$ and $z_{1}=z_{2}=0$ are invariant systems of equations. But $z_{2}=0$ is a consequence of $z_{1}=0$, which gives no new results. These are all the invariant equations of an order lower than the third.

If we extend the transformations three times, we have the matrix

$\left\|\begin{array}{|ccccccccc}1 & 0 & 0 & 0 & 0 & 0 & 0 & 0 & 0 \\ 0 & 1 & 0 & 0 & 0 & 0 & 0 & 0 & 0 \\ 0 & 0 & 1 & 0 & 0 & 0 & 0 & 0 & 0 \\ 0 & x & 0 & 1 & 0 & 0 & 0 & 0 & 0 \\ y & 0 & 0 & -y_{1}^{2} & -y_{1} z_{1} & -3 y_{1} y_{2}-\left(y_{2} z_{1}+2 y_{1} y_{2}\right) & -\left(4 y_{1} y_{3}+3 y_{2}^{2}\right)-\left(y_{3} z_{1}+3 y_{2} z_{2}+3 y_{1} z_{3}\right) \\ 0 & 0 & z & 0 & z_{1} & 0 & z_{2} & 0 & z_{3} \\ x & -y & 0 & -2 y_{1} & -z_{1} & -3 y_{2} & -2 z_{2} & -4 y_{3} & -3 z_{3} \\ 0 & 0 & z^{2} & 0 & 2 z_{1} & 0 & 2\left(z_{1}{ }^{2}+z_{2}\right) & 0 & b z_{1} z_{2}+2 z_{3}\end{array}\right\|$


WILLIAMS. ON THE GEOMETRI OF THE TRANSFOMMATION GROUP, ETC.

Indicating, in this case, by $d_{t}$ the determinant formed by suppressing the ith column, we find

$$
\begin{aligned}
& \lrcorner_{1}-18 z_{1}^{3} y_{2}^{3}, \quad J_{s}=3 z_{1}^{4} y_{2} y_{3}, \quad J_{7}=18 z_{1}{ }^{3} y_{2}^{3} z_{2}, \\
& J_{\mathrm{i}} \quad 2 z_{1}\left(6 y_{2} z_{3} z_{1}-4 z_{1}{ }^{2} y_{3}{ }^{2}-9 y_{2}{ }^{2} z_{2}{ }^{2}\right), \quad J_{0}: \quad 18 z_{1} y_{2}\left(y_{2}^{2} z_{1} z_{3}-3 z_{2}{ }^{2} y_{2}{ }^{2}\right) .
\end{aligned}
$$

The others vanish identically. We see from these determinants that the only single invariant equation is $z_{1}=0$, while $z_{1}=y_{2}=0$ is an invariant system; and it is clear that these are the only results obtained from further extension of the transformations. Hence, all invariant equations of the third and higher orders are obtained from the differential invariants.

By writing $J_{3}=0$, we tiud in particular under this head $y_{2}=y_{3}=0$ as an invariant system; but as $y_{3}=0$ is a consequence of $y_{2}=0$, this case gives us nothing new.

I : If now we perform the transformations of the $G_{\mathrm{s}}$ on a curve which admits of no transformation of the $G_{s}$, this curve generates an invariant family of $\infty^{5}$ curves. They may be represented by:

(a) One equation of the zero order and one of the VIII. 0.

(b) One equation of the $I .0$. and one of the $V I I .0$.

(c) One equation of the $I I .0$. and one of the $V I .0$.

(d) One equation of the $I I I .0$. and one of the $V .0$.

(e) Two equations of the $/ V$. 0 .

Since no equation of the zero order exists, case (a) is excluded.

ln considering case (b), we find that the only differential equation of the $I .0$. is $z_{1}=0$. Hence the curves are plane curves, lying in the $\sigma^{\text {' planes }}$ $\approx=$ const. Since no figure in the $x y$-plane is changed by $r, z r, z^{2} r$, the $\infty^{7}$ curves are the same in each plane parallel to the $x y$-plane. Hence the differential equation of the $V I I .0$. is one which is invariant under

of which the form is

$$
p, \quad q, \quad y p, \quad x p-y \llbracket, \quad x q
$$

$$
H^{\prime}\left(J_{4} \ldots J_{i}\right)=0 \text {. }
$$

Lie has shown how to reduce this equation in the following mauner.

It is easily seen that it can be written in the form

$$
F\left[S_{4} J_{5} \frac{d J_{5}}{d J_{4}} \frac{d J^{2} J_{5}}{d J_{4}^{2}}\right]=0
$$

where

$$
J_{4}=y_{2}^{-\frac{1}{3} \sigma_{2}}, \quad \delta_{5}=\dot{y}_{2}^{-1} \omega_{3},
$$

and $\quad i_{2}=3 y_{2} y_{4}-5 y_{3}^{2}, \quad i_{3}=3 y_{2}^{2} y_{5}-15 y_{2} y_{3} y_{4}+\frac{40}{3} y_{3}^{3}$.

If (2) has been integrated we find an equation of the general form

$$
J_{5}=\phi\left(J_{1}\right) \text {. }
$$


Now introduce as new variables $J_{4}$, and $U=y_{2}^{-1} y_{3}$; then we find

or

$$
\frac{d U}{d J_{4}}=\frac{1}{3} \frac{\rho_{2}+y_{3}^{2}}{y_{2}^{2} / /_{3}}=\frac{1}{3} \frac{J_{4}+U^{2}}{J_{5}}
$$

which is a Riccati's equation.

$$
\frac{d U}{d J_{4}}=\frac{1}{3} J_{4}+U^{2}
$$

If

$$
W\left(U J_{4}\right)=\text { const. }
$$

is an integral equation of (3), we can find two other integral equations o $J_{i}=\Phi\left(J_{1}\right)$ as follows. Extending $y p$, we have

$$
X f=y \frac{\partial f}{\partial x}-y_{1}^{2} \frac{\partial f}{\partial y_{1}}-3 y_{1} y_{2} \frac{\partial f}{\partial y_{2}}-\left(4 y_{1} y_{3}+3 y_{2}^{2}\right) \frac{\partial f}{\partial y_{3}}-\left(5 y_{1} y_{4}+10 y_{2} y_{3}\right) \frac{\partial f}{\partial y_{4}}
$$

from which we see that

$$
X\left(J_{4}\right)=0, \quad X(U)=-3 y_{2}^{3}
$$

and

$$
\begin{aligned}
& X(W)=-3 \frac{\partial}{\partial} W^{r}{ }^{2} \\
& X X(W)=9 y_{2} \frac{\partial^{2} W}{\partial U^{2}}+6 y_{i} y_{2}^{: 3} \frac{\partial W}{c} .
\end{aligned}
$$

Now

$$
W=\text { const., } \quad X(W)=\text { const., } \quad X X(W)=\text { const. }
$$

represent three independent integral equations of $J_{i j}=\mathscr{J}\left(J_{4}\right)$; and if we eliminate $y_{;}, y_{4}, y_{3}$, we obtain a differential equation in $y_{1}$ and $y_{3}$, which can be integrated by two quadratures.

For case (c) we know that the only differential equation of the $I T .0$. is found by writing $I_{3}=\infty$, which gives

$$
y_{2}=0 \text {. }
$$

But then all the difterential invariants of higher orders either vanish or become infinite; so that this case is exclucled.

The two equations under (d), of the $I I I .0$. and $V .0$. are

$$
I_{3}-\frac{9 y_{2}^{2} z_{2}^{2}+z_{1}\left(z_{1} y_{3}^{2}-6 y_{2}^{2} z_{3}\right)}{z_{1}^{2} y_{2}^{8 / 3}}=\text { const. }
$$

and

$$
F\left(J_{4}, J_{5}\right)=0 \text {. }
$$

The integration of (2) can be reduced as in case (b). If from $H^{\top}=0$ we find $y=\varphi(x)$, then, substituting in (1) for $y_{2}$ and $y_{3}$ their values in terms of $x$, we have

$$
I_{3}-\frac{9 \varphi_{2} z_{2}^{2} z^{2}+z_{1}\left(z_{1} \varphi_{3}{ }^{2}-6 \varphi_{2}{ }^{2} z_{3}\right)}{z_{1}^{2} \varphi_{2}{ }^{2}{ }^{37}}=\text { const., }
$$


WILLIAMS. ON THE GEOMETIY OF THE TRANSFORMATION GROUP, ETC.

which is a differential equation of the third order in $z$ and $x$. This equation may be written in the form

$$
\frac{z_{3}}{z_{1}}-\frac{3}{2} \frac{z_{z}^{2}}{z_{1}^{2}}=F(x)
$$

which, as Lie has shown, can be reduced to a Riccati's equation of the first order.

If we put

$$
\frac{z_{2}}{z_{1}}=y
$$

then will

$$
\frac{d y}{d x}=\frac{z_{3}}{z_{1}}-\frac{z_{2}^{2}}{z_{1}^{2}}
$$

or

$$
\frac{d y}{d x}=1 / 2 y^{2}+F(x) \text {. }
$$

If $W(y x)=$ const. is an integral equation of this Riccati's equation, we can find the required integral equations of $v=F(x)$ by differentiation. If we write

$$
X_{x} f^{\prime} \quad z^{2} \frac{\partial f}{\partial z}+2 z z_{1} \frac{\partial f}{\partial z_{1}}+\left(2 z z_{2}+2 z_{1}^{2}\right) \frac{\partial f^{\prime}}{\partial z_{2}}
$$

then $X_{x} W=$ const. and $X_{x}\left(X_{s} W\right)=$ const. are known integral equations of $w=F(x)$; it is sufficient, therefore, to show that $W, X_{s} W^{\prime}$, and $X_{s}\left(X_{s} W\right)$ are independent functions of $x, z, z_{1}$ and $z_{2}$. From

$$
\begin{aligned}
X_{*}(x) & =0 \\
X_{s} W & =\frac{\hat{\partial} W}{\partial y} X_{s}(y)=2 \frac{\hat{\partial} W}{\partial y} z_{1} \\
X_{s}\left(X_{s} W\right) & =4 \frac{\partial^{2} W^{r}}{\partial y^{2}} z_{1}^{2}+4 \frac{\partial W}{\partial y} z z_{1}
\end{aligned}
$$

we see that $\mathbb{W}^{r}, X_{s} W, X_{s}\left(X_{s} W\right)$ are really independent with respect to $z, x, z_{1}$, and $y$. Thus the integration of $w=F(x)$ is rednced to the Riccati's equation.

Finally, considering case (e), we see that if the differential invariants are $I_{3}, I_{4}, J_{4}$, the two equations of the fourth order may be written

or

$$
\mathscr{L}_{1}\left(I_{3}, I_{4}, J_{4}\right)=0, \quad \varrho_{2}\left(I_{3}, I_{4}, J_{4}\right)=0
$$

$$
\Phi\left(I_{3}, I_{1}\right)=0, \quad \Psi\left(I_{3}, J_{1}\right)=0 \text {. }
$$

II: If now the curve admits of one transformation of the $G_{8}$, the invariant family consists of only $\infty^{7}$ curves, which are defined

(a) By one equation $I .0$. and one VI.0.; which are $\Sigma_{1}=0, F\left(J_{1} \ldots J_{0}\right)$ $=0$. This case is similar to case (b) in $\mathrm{I}$.

(b) By one equation $I T .0$. and one $V .0$; which case, as in $I$, is excluded. 
24 WILLIAMS. ON THE GEOMETRY OF THE TRANSFOMMATION GROUP, ETC.

(c) By one equation $I I I$. 0. and one $I V .0$. They are clearly $I_{3}=$ const. and $J_{1}=$ const. The second equation can be integrated. The first can also be expressed in the variables $x$ and $z$ as in case (d) in I.

III. If the curve admits of two transformations, the family consists of $\infty{ }^{6}$ curves, which are defined

(a) By one equation $I .0$. and one $V .0$.

They are $z_{1}=0$ and $F\left(J_{t}, J_{i 5}\right)=0$; and this case is similar to (a) in II.

(b) By one equation of the $I I .0$. and one of the $I V .0$.

'This case is agrain excluded.

(c) By two equations of $I I I .0$.

Since there is only one invariant equation of the third order, we exclude this case also.

IV. If the carve almits of three trausformations there are $\infty^{5}$ curves in the family, which are represented by

(a) One equation of the $I .0$. and one of the $I V$. 0 .

(b) One equation of the $I I .0$. and one of the $I I I .0$.

Cases similar to (a) have already been considered. Case (b) gives only $y_{2}=.0$ as the invariant equation of the second order. The only invariant system would be $y_{2}=0, y_{3}=0$, which is excluded. For $y_{3}=0$ is a consequence of $y_{2}=0$; that is the integration of $y_{3}=0$ must give $y_{2}=0$; otherwise the equations are incompatible. So that we have only one invariant equation.

V. Suppose next that the curve admits of four transformations of the $G_{*}$; the family then consists of $\infty{ }^{\prime}$ curves, and is defined by

(a) One equation of the $l .0$. and one of the $I I T .0$.

(b) Two equations of the $I I .0$.

Case (a) is excluded ; for if $z_{1}=0, I_{3}$ becomes elusive. Also case (b) is excluded, since there do not exist two invariant equations of the second order.

VI. If the curve admits of five transformations, the $\infty^{3}$ curves of the family then generated ire defined by

(a) One equation $I .0$. and one $I I .0$.

As we have seen, the equation of the first order is $z_{1}=0$. There are two of the second order which we can consider in comnection with $a_{1}=0$. They are $y_{2}=0$ and $z_{2}=0$. If we take $y_{2}=0$ and $z_{1}=0$, we find $\infty^{3}$ straight lines lying in the $\infty^{1}$ planes $z=$ const. But $z_{1}=0$ and $z_{2}=0$ give us no case, since $z_{z}=0$ is a consequence of $z_{1}=0$.

VII. If the curve almits of six trausformations of the group, the $s$ " curves of the family must be defined by two invariant equations of the first order. But since there is only one invariant of the first order, this cisse is excluded. 
WILLIAMS. ON THE GEOMETRY OF THE TRANSFORMATION GROUP, ETC.

VIII. Lastly we consider the case when the curve admits of seven transformations. But this case is excluded, since the $\infty^{1}$ curves of the family would have to be defined by an invariant equation of the zero order in connection with one of the first order, while no invariant equation of the zero order exists.

From the consideration of all these cases we conclude that there are no invariant families of $\infty^{4}, \infty^{2}$, or $\infty^{1}$ curves. In other words, no curve admits of exactly four or exactly six or exactly seven transformations of the $G_{s}$. Hence, if two curves are equivalent under the $G_{s}$, they are either members of families of plaue curves defined by $F\left(J_{4} \ldots J_{k}\right)=0$, lying in the planes $z=$ const.; or they are nembers of a family $\infty^{s}$ curves defined by $I_{3}=$ const. and $F\left(J_{1}, I_{5}\right)=0$, or $\Phi\left(I_{3} I_{4}\right)=0$ and $W\left(I_{3} J_{4}\right)=0$; or finally they are members of a family of $\infty^{7}$ curves defined by $I_{3}=$ const. and $I_{4}=$ const.

\section{$\$ 4$.}

10. Haviug disposed of the problem concerning invariant curve-families as defined by invariant differential equations, the next step which most naturally suggests itself is the corresponding investigation for invariant families of suifuces. We shall accordingly devote this section to finding all the differential invariants which express properties of surfaces, and invariant partial differeutial equatious.

Here $z$ must be considered as some function of $x$ and $y$, say

We shall in general write

$$
z=f(m y) \text {. }
$$

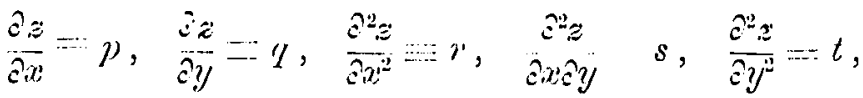

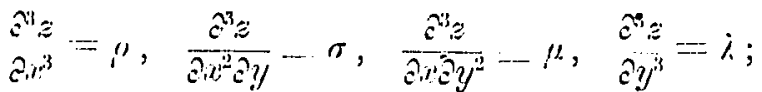

and fiud in the following manner the increments receired by the functions $p$, y, etc., uncler the transformations of the $G_{*}$.

If then

we have

$$
z=f(x y)
$$

and hence

$$
d z=p d x+q d y,
$$

$$
d i z=d p \cdot d x+d q \cdot d y+p \cdot d x x+q \cdot d b y .
$$

In extending the transformations of the group by means of (1) we shall, as usual, preserve the order in which they occur in Section I.

. Tt is clear that $\partial p=\partial q=0$ for $X_{1} f, X_{1} f$, and $X_{3} f$. 
WILLLALS. ON 'THE GEOMETRY OF 'THE TRANSFORMITION GROUR, ELC.

For $X_{i} f$, since $i z=0$, we have

$$
0 \quad \partial p \cdot d x+\partial q \cdot d y+q d x=(i p+q) d x+i z \cdot d y
$$

from which we tind

$$
\partial p=-q, \quad \partial_{q} \quad 0 \text { : }
$$

In the same way we see that for $X_{5_{0}} f$

$$
i p-0, \quad \partial y--p \text {. }
$$

For $X_{60} f$ we have $i z-z$. Hence

or

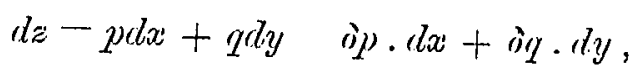

which shows that

$$
(\delta p-p) d x+(j q-q) \dot{d} y=0
$$

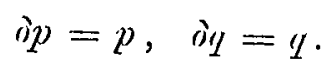

In exactly the same way the increments of $p$ and $q$ for $X_{i_{0}} f$ and $Y_{s} f$, respectively, are found to be

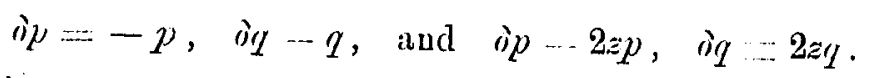

Hence tho trausformations once extended may be written

$$
\begin{aligned}
& X_{1} f=\frac{\partial f}{\partial x} \\
& x_{2}+\cdots=\frac{\partial f}{\partial y} \\
& \mathrm{X}_{3} t=\frac{\partial t}{\partial z} \\
& X_{t} t=x \frac{\partial f^{\prime}}{\partial y}-g \frac{\partial f}{\partial p} \\
& X_{0} t-y \frac{\partial f}{\partial x}-p \frac{\partial f}{\partial q} \\
& X_{t_{t}} f-z \frac{\partial f}{\partial z}+p \frac{\partial f}{\partial p}+q \frac{\partial f}{\partial q} \\
& X_{i r} f-x \frac{\partial f}{\partial x}-y \frac{\partial f}{\partial y}-p \frac{\partial f}{\partial p}+q \frac{\partial f}{\partial q} \\
& X_{\mathrm{s}} f-z^{2} \frac{\partial f}{\partial z}+2 z p \frac{\partial f}{\partial p}+2 z q \frac{\partial f}{\partial q} .
\end{aligned}
$$

To write $Y_{1} f^{\prime}=0 \ldots X_{q} f^{2}=0$ would give no result, since then the first three equations wonld show that the invariant function of would have to be free of $x, y, z$ and consequently the others would show that it would have to be free of $p$ and $q$ also.

Let us then extend the transformations again. We know that

$$
d p \quad r d x+s d y, \quad d y-s d x+t d y
$$


WILLIANS. ON THE GEOMETRY OF 'IHE T'RANSFORMATION GROUP, ETC.

so that

$$
\begin{aligned}
& d i p-\partial r \cdot d x+d s . d y+r . d i x+s . d i y\}
\end{aligned}
$$

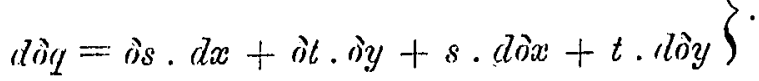

It is again clear from (2) that $p$, $q$, etc., receive no increments for $X_{l_{e}} f$, $X_{2} f$, and $X_{3_{1}} f$.

For $d_{t} f$,

$$
i p=-\eta
$$

hence

$$
-d q-(s d x+t d y)-i r \cdot d x+i s \cdot d y+s d x
$$

from which we find

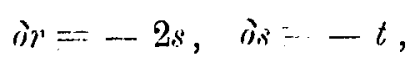

while the second of equations (2) shows that $\partial t=0$.

In precisely the same manuer $i r$, is, iit can be found for the other transformations, and the complete system of eight members in eight variables may be written

$$
\begin{aligned}
& \dot{x_{i}} f-\frac{\partial f}{\dot{c} x}=0 \\
& X_{x}+\quad \frac{\partial f}{\partial y}=0 \\
& X_{0} f^{\prime}-\frac{\partial f}{\partial z}=0 \\
& X_{1} f-x \frac{\partial f}{\partial y}-\eta \frac{\partial f}{\partial p}-2 s \frac{\partial f}{\partial r}-t \frac{\partial f}{\partial s}=0 \\
& x_{i r} f-y \frac{\partial f}{\partial x}-y \frac{\partial f}{\partial q}-r \frac{\partial f}{\partial s}-2 s \frac{\partial f}{\partial t}=0 \\
& I_{t} f-z \frac{\partial f}{\partial z}+p \frac{\partial f}{\partial p}+q \frac{\partial f}{\partial q}+r \frac{\partial f}{\partial r}+s \frac{\partial f}{\partial s}+t \frac{\partial f}{\partial t}=0 \\
& I_{7} f=x \frac{\partial f}{\partial x}-y \frac{\partial j}{\partial y}-p \frac{\partial j}{\partial p}+y \frac{\partial j}{\partial q}-2 r \frac{\partial f}{\partial r}+2 t \frac{\partial f}{\partial t}=0 \\
& X_{s} f \quad z^{2} \frac{\partial \vec{f}}{\partial z}+2 z p \frac{\partial f}{\partial p}+2 z q \frac{\partial f}{\partial q}+2\left(z r+p^{2}\right) \frac{\partial f}{\partial r}+(2 z s+p q) \frac{\partial f}{\partial s} \\
& +2\left(\approx t+q^{2}\right) \frac{\partial t}{\partial t}=0 \text {. }
\end{aligned}
$$

Since $x, y, z$ are not contained in $f$, the determinant of these equations may, after an obvious reduction, be written

$$
\left|\begin{array}{ccccc}
-q & 0 & -2 s & -t & 0 \\
0 & -p & 0 & -r & -2 s \\
\eta & q & r & s & t \\
-p & q & -2 r & 0 & 2 t \\
0 & 0 & p^{2} & p q & q^{2}
\end{array}\right| .
$$


WILLIANS. ON 'IHE GEOMETRY OF THE IRANSFOMMATION GROUP, E'TC.

If we expand this determinant and place the result equal to zero, we have an invariant differential equation.*

The equation is easily found to be

It has for its integral

$$
p^{2} t-2 p q s+q^{2}{ }^{2}=0 .
$$

or

$$
\begin{gathered}
y=x \varphi(a)+\psi(a), \quad z=a, \\
y=x \varphi(z)+\phi(z),
\end{gathered}
$$

which is the general equation to ruled surfaces, where the straight line generators are all parallel to the $x y$-plane.t

11. Since the determinant considered above is not identically zero, the complete system has no solution. In order then to have more variables than equations, and therefore one or more solutions, it is necessary to extend the trausformations further. To do this we have

$$
\begin{aligned}
& d r=\sigma d x+\sigma d y \\
& d s-\sigma d x+\mu d y \\
& d t \equiv \mu d x+\lambda d y ;
\end{aligned}
$$

also

$$
\begin{aligned}
& d \partial r-\partial u \cdot d x+\partial \sigma \cdot d y+a \cdot d \dot{\prime} x+\sigma . d i y) \\
& d \lambda s=\partial \sigma \cdot d x+\partial \mu \cdot d y+\sigma \cdot d \partial x+\mu \cdot d \partial y\} . \\
& d \partial t: \partial \mu . d x+\partial \lambda . d y+\mu . d \partial x+\lambda . d \partial y \mid
\end{aligned}
$$

Here again $p$, $q$, etc., receive no increments under $X_{1} f, X_{2} f$, and $X_{3} f$.

Since for $X_{4} f$, ix -0 , ì $x$, ip - $q$, iq -0 , ir $-2 s$, is $-t$, $i t=0$; we have from $(3)$

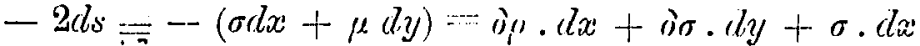

$$
\begin{aligned}
& -d t-(\mu d x+\dot{\lambda} d y)=i \sigma \cdot d x+i \mu \cdot d y+\mu d x \\
& 0 \text { - } 0 \quad . j p . d x+i j . d y+\lambda . d x .
\end{aligned}
$$

If in these last three equations we collect the coefficients of $d x$ and $d y$ and equate separately to zero, we find

$$
\partial_{0}--3 \sigma, \quad \partial \sigma=-2 \mu, \quad \partial \mu \cdots-\lambda, \quad j \lambda-0 .
$$

For $X_{5} f$, equations (3) have the forms

$$
\begin{aligned}
& 0-\partial_{1} \cdot d x+i \sigma \cdot d y+o \cdot d y \\
& -d r \ldots-(o d x+\sigma d y)=j \sigma \cdot d x+\partial \mu \cdot d y+\sigma \cdot d y \\
& -2 d s=-2(\sigma \cdot d x+\mu d y) \quad \partial \rho \alpha \cdot d x+i d \cdot \cdot d y+\mu \cdot d y ;
\end{aligned}
$$

from which we tind

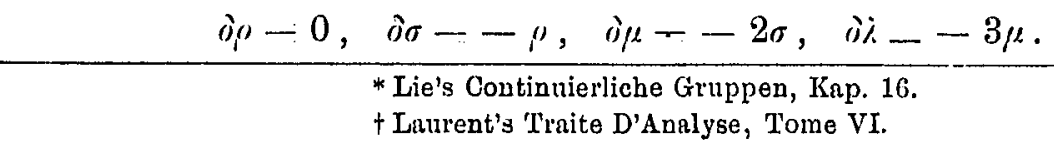


WILLIAMS. ON THE GEOMETRY OF THE TRANSTORMATION GROUP, ETC.

In like manner it will be easily seen that for $X_{6_{r}} f^{\prime}$

For $X_{i} f$

$$
i_{i} \ldots, \quad \nu, \quad i \sigma \ldots, \quad i \mu \equiv \mu, \quad \partial i \equiv \lambda .
$$

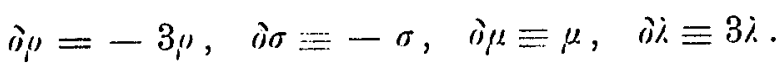

Finally we have for $X_{S_{1}} f$

$$
\begin{aligned}
& \lambda_{\prime \prime} \equiv 2 z_{1}^{\prime}+6 \eta p, \quad i \sigma \equiv 2 z \sigma+2 \eta \eta+4 p s \\
& i \eta \equiv 2 z \eta+2 p t+4 \eta s, \quad i \lambda \equiv 2 z \lambda+6 q t .
\end{aligned}
$$

If now the trinsformations be written in the extended form, and $\Gamma_{s} f$ be replaced by $X_{s} f^{\prime}-2 X_{i} f^{\prime}$; and if the terms contining $\frac{\partial f}{\partial x}, \frac{\partial f}{\partial y}, \frac{\partial f}{\partial z}$ be omitted (since $x, y$, and $z$ do not occur in the solutions) the complete system may be written in the form

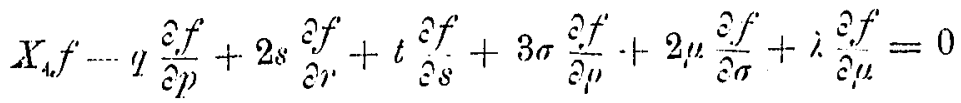

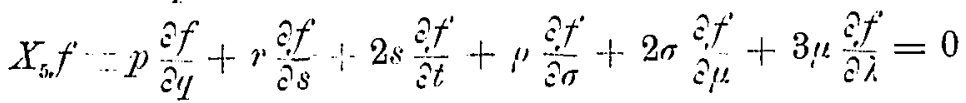

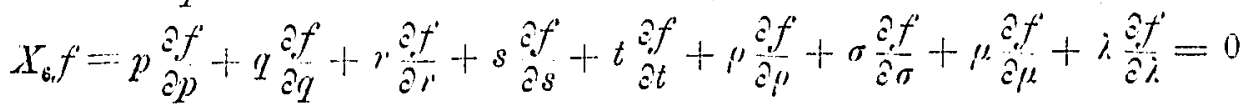

$$
\begin{aligned}
& X_{7} f-p \frac{\partial f}{\partial p}+q \frac{\partial f}{\partial q}-2 r \frac{\partial f}{\partial r}-2 t \frac{\partial f^{\prime}}{\partial t}-3{ }^{\prime \prime} \frac{\partial f}{\partial f^{\prime}}-\sigma \frac{\partial f}{\partial \sigma}+\mu \frac{\partial f}{\partial f^{\prime}}+3 i \frac{\partial f^{\prime}}{\partial x}=0 \\
& X_{s} f=p^{2} \frac{\partial f}{\partial r}+p q \frac{\partial f}{\partial s}+q^{2} \frac{\partial f}{\partial t}+3 r p \frac{\partial f}{\partial_{\prime \prime \prime}^{\prime \prime}}+(r q+2 p s) \frac{\partial f}{\partial \sigma}+(p t+2 q s) \frac{\partial f}{\partial f^{\prime}} \\
& +3 p t \hat{c} \hat{c} \hat{c}^{\prime}=0 \text {. }
\end{aligned}
$$

The next object is to find the solutions of this complete system ; and owing to the complicated forms in which some of the results occur, we shall resort to a device by means of which the work can be greatly simplified.

Let us first consider the complete system given by the sub-group

$$
\begin{aligned}
& X_{t} f-q \frac{\partial f}{\partial p}+2 s \frac{\partial f}{\partial f}+t \frac{\partial f}{\partial t}=0 \\
& X_{s t} f-p \frac{\partial f}{\partial t}+r \frac{\partial f}{\partial s}+2 s \frac{\partial f}{\partial t}=0 \\
& X_{i t} f=-p \frac{\partial f}{\partial p}+q \frac{\partial f}{\partial t}-2 r \frac{\partial f}{\partial r}+2 \frac{\partial f}{\partial t}=0 .
\end{aligned}
$$

The solntions of $X_{7} f=0$ are found to be

$$
\text { * } u_{1}, \quad p g=u_{2}, \frac{p^{2}}{r} u_{3}, \quad p^{2 i} \cdots u_{1} .
$$


WILLIAMS. ON THE GEOME'TRY OF THE TRANSFORMATION GROUP, ETC.

These solutions can now be introduced into the other two equations in the usual way. It will be seen that

$$
\begin{aligned}
& X_{1}\left(u_{1}\right)=t-\frac{u_{4}}{p^{2}} \\
& X_{1}\left(u_{3}\right)-\tau^{2}-\frac{u_{2}^{2}}{p^{2}} \\
& X_{1}\left(u_{3}\right)=\frac{2 p q}{r}-2 s\left[-\frac{p^{2}}{p^{2}}\right)-\frac{2}{p^{2}}\left(u_{2} u_{3}-u_{1} u_{3}{ }^{2}\right) \\
& X_{1}\left(u_{1}\right)-2 p q t-\frac{2}{p^{2}} u_{2} u_{1} .
\end{aligned}
$$

In like manner it will be found that

$$
X_{5}\left(u_{i}\right)-\frac{p^{2}}{\varphi\left(u_{i}\right)}
$$

and the two equations will have the forms

$$
\begin{aligned}
& X_{4_{2}} r=u_{4} \frac{\partial f}{\partial u_{1}}+u_{2}{ }^{2} \frac{\partial f}{\partial u_{2}}+2\left(u_{2} u_{3}-u_{1} u_{3}{ }^{2}\right) \frac{\partial f}{\partial u_{3}}+2 u_{2} u_{4} \frac{\partial f}{\partial u_{4}}=0 \\
& X_{5} f^{\prime}=\frac{1}{u_{3}} \frac{\partial f}{\partial u_{1}}+\frac{\partial f}{\partial u_{2}}+2 u_{1} \frac{\partial f}{\partial u_{4}}=0 .
\end{aligned}
$$

The solutions of $X_{n} f=0$ are

$$
u_{3} \quad v_{1}, \quad u_{1} u_{3}-u_{2}-v_{3}, \quad u_{1}^{2} u_{3}-u_{4} \quad v_{3} .
$$

When these solutions are introduced, $X_{4,} f$ has the form

$$
X_{4} f=2 v_{1} v_{2} \frac{\partial f}{\partial v_{1}}+\left(v_{2}^{2}+v_{1} v_{3}\right) \frac{\partial f}{\partial v_{2}}+2 v_{2} v_{3} \frac{\partial f}{\partial v_{3}}=0
$$

and this equation is equivalent to

$$
\frac{d v_{1}}{2 v_{1} v_{2}}=\frac{d v_{2}}{v_{2}^{2}+v_{1} v_{3}}=\frac{d v_{3}}{2 v_{2} v_{3}}
$$

One solution is clearly $\frac{v_{3}}{v_{1}}$, aud, if we place this equal to some constant $a$, we have in order to determine the other

$$
\frac{d v_{1}}{2 v_{1} v_{2}}=\frac{d v_{2}}{v_{2}^{2}+a v_{1}^{2}}
$$

This equation is homogeneous, and it is therefore known at once that the integrating factor is

$$
-\frac{1}{v_{2}^{2} \cdot v_{1}-a v_{1}^{3}}{ }^{*} *
$$

* Page's Ordinary Differential Equations, Art. 64. 
WILLIAMS. ON THE GEOMETRY OF THE TRANSFORMATION GROUP, ETC.

Hence we have

$$
\int \frac{2 v_{1} v_{2}}{v_{1} v_{2}^{2}-a v_{1}^{3}} d v_{2}+\int\left[-\frac{v_{2}^{2}+a v_{1}^{2}}{v_{1} v_{2}^{2}-a v_{1}^{3}}-\frac{v_{2}^{2}-3 a v_{1}^{2}}{v_{2}^{2} v_{1}-a v_{1}^{3}}\right] d v_{1}=c
$$

and therefore

$$
\log \left(v_{1} v_{2}^{2}-a v_{1}^{3}\right)-\log v_{1}^{2}=c
$$

The two solutions are then

$$
\frac{v_{3}}{v_{1}}=u_{1}^{2}-\frac{u_{4}}{u_{3}} \quad s^{2}-r t
$$

and

$$
\frac{v_{1} v_{2}^{2}-a v_{1}^{3}}{v_{1}^{2}}-\frac{v_{2}^{2}-v_{1} v_{3}}{v_{1}}+q^{2}-2 p q s+p^{2} t
$$

12. We shall next seek differential par'ameters for this sulb-group by means of which all solutions of a higher order can be found. We know that if $\varphi$ is a function of $x$ and $y$

$$
d \varphi=\frac{\hat{c} \varphi}{\hat{\imath} x} d x+\frac{\hat{c} \varphi}{\hat{c} y} d y
$$

or writing $\frac{\dot{c} \varphi}{\partial x}-\varphi_{x}$, and $\begin{aligned} & \hat{c} \varphi \\ & \hat{\varepsilon} y\end{aligned}-\varphi_{y}$,

$$
d \varphi=\varphi_{x} d x+\varphi_{y} d y \text {. }
$$

Also

$$
d \partial y=i c_{x} \cdot d x+i c_{y} \cdot d y+\varphi_{x} \cdot d i x+\varphi_{y} \cdot d x y .
$$

If now $\varphi$ is to be an invaricant function, then must $\rho_{\varphi}=0$; and therefore,

$$
0=i \varphi_{x} \cdot d x+i \varphi_{y} \cdot d y+\varphi_{x} \cdot d i x+\varphi_{y} \cdot d i y .
$$

For $X_{4} f^{\prime}=x q$, equation (4) becomes

$$
0=i \varphi_{x} \cdot d x+i \varphi_{y} \cdot d y+\varphi_{y} \cdot d x-\left(r c_{x}+\varphi_{y}\right) d x+i \varphi_{y} \cdot d y
$$

from which we gret

$$
\lambda \varphi_{x}=-\varphi_{y}, \quad i \varphi_{y}=0
$$

In like manner for $X_{i_{1}} f$ it is easily seen that

and for $X_{7} f$

$$
\lambda \varphi_{x}=0, \quad \dot{s}, n=-\varphi_{x}
$$

$$
\partial \varphi_{x}=-\varphi_{x}, \quad i \varphi_{y}=\varphi_{y} .
$$

Hence the complete system lias the form

$$
\begin{aligned}
& X_{u_{1}} f \ldots q \frac{\partial f}{\partial p}+2 s \frac{\partial f}{\partial r}+t \frac{\partial f}{\partial s}+\varphi_{1 y} \frac{\partial f}{\partial \varphi_{x}}=0 \\
& X_{s_{1}} f-p \frac{\partial f}{\partial q}+r \frac{\partial f}{\partial s}+2 s \frac{\partial f}{\partial t}+\varphi_{x} \frac{\hat{\partial} f}{\partial \varphi_{y}}=0 \\
& X_{y} f=-p \frac{\partial f}{\partial \eta}+q \frac{\partial f}{\partial \eta}-2 v \frac{\partial f}{\partial r}+2 t \frac{\partial f}{\partial t}-\varphi_{x} \frac{\partial f}{\partial \varphi_{x}}+\varphi_{y} \frac{\partial f}{\partial \varphi_{y}}=0 \text {. }
\end{aligned}
$$


We already know two solutions of this complete system. If now we solve $I_{i,} f=0$, we find among other solutions

$$
J_{1} \varphi \cdots \cdots \varphi_{y}-q \varphi_{x} .
$$

This solution is fond to be common to the other two equations also. Again it is observed that $\varphi_{x}$ and $\varphi_{y}$ enter the three equations just as do $p$ and $q$, respectively. We can then write the other solution at once, which is

$$
\nu_{1} \varphi \cdot \varphi_{x}{ }^{2} t-2 \varphi_{x} \varphi_{y} s+\varphi_{y}{ }^{2} r
$$

By meaus of the differential parameters, $\vec{J}_{1} \varphi$ and $D_{1} \varphi$, can be found all solutions of higher order common to $X_{r_{i}} f=0, Y_{r_{i}} f=0$, and $X_{i} f=0$. We can then introduce these solntions into $X_{6} f=0$ and $X_{s i} f=0$, and thus find the solutions of our original complete system. We shall be content with finding one of these solutions and a differential parameter for the whole group.

One of the solutions common to $X_{1,} f=0, X_{s_{n}} f=0$, and $X_{i} f=0$, was found to be

$$
p^{2} t-2 \eta q s+q^{2} r-u_{1} \text {. }
$$

When the variables $", \sigma, \mu$, and $i$ are added, another can be found by means of $J_{1} c$, which has the form

$$
J_{1}\left(u_{1}\right)-p^{3} i-3 p^{2} \gamma p^{2}+3 p q^{2} \sigma-q_{1}^{3} \cdots-u_{2} .
$$

If now $u_{1}$ and $u_{2}$ be introduced into $X_{s} f=0$, it is easily seen that they are solutions of $X_{i f} f=0$ also. We then introduce the same into $Y_{i j} f=0$, and find

which has the solntion

$$
X_{i_{1}} f^{\prime} \quad 3 u_{1} \frac{\partial f}{\partial u_{1}}+4 u_{2} \frac{\partial f^{\prime}}{\partial u_{u}}=0
$$

$$
\frac{u_{1}^{1}}{u_{2}^{3}} \frac{\left(p^{2} t-2 p q s+q^{2} r\right)^{4}}{\left(p^{3} i-3 p^{2} q q^{2}+3 p q^{2} \sigma-q^{3}{ }^{3}\right)}-I_{3}
$$

The other three could be found in a similar manner. We will say they are $J_{3}, h_{3}$, and $L_{3}$.

13. We shall next find differential parameters for the complete system.

It is clear that for $X_{i i} f$ and $X_{s} f$

$$
\partial \varphi_{x}=\partial \varphi_{y}=0 .
$$

If now we introduce into $X_{i} f^{\prime}=0$ and $X_{s_{t}} f^{\prime}=0$ the solutions

$$
\begin{aligned}
& s^{2}-r t-u_{1} \\
& p^{2} t-2 p q^{s}+q^{2} r \cdots u_{2} \\
& p \varphi_{y}-q \varphi_{x} u_{3} \\
& \varphi_{x}{ }^{2} t-2 \varphi_{x} \varphi_{y} s+\varphi_{y}{ }^{2} r=u_{1},
\end{aligned}
$$


WILLIANSS. ON THE GEOMETRY OF THE TRANSFOMMATION GROUP, ETC.

the two equations take the forms

$$
\begin{aligned}
& X_{6_{1}} f-2 u_{1} \frac{\partial f}{\partial u_{1}}+3 u_{2} \frac{\partial f}{\partial u_{2}}+u_{3} \frac{\partial f}{\partial u_{3}}+u_{4} \frac{\partial f}{\partial u_{1}}=0 \\
& X_{8} f \cdots-u_{2} \frac{\partial f}{\partial u_{1}}+u_{3}{ }^{2} \frac{\partial f}{\partial u_{4}}=0 .
\end{aligned}
$$

The solutions of $I_{s} f=0$ are

$$
u_{2}=v_{1}, \quad u_{3}-v_{2}, \quad u_{1} u_{3}^{2}+u_{2} u_{4} \quad v_{3} .
$$

When these solutions are introduced, $X_{t_{t}} f$ has the form

$$
X_{0} f=3 v_{1} \frac{\partial f}{\partial v_{1}}+v_{2} \frac{\partial f}{\partial v_{3}}+4 v_{3} \frac{\partial f}{\partial v_{3}}=0
$$

The solutions are easily found to be

and

$$
J_{1} \varphi \equiv \frac{v_{2}^{3}}{v_{1}} \equiv \frac{u_{3}^{3}}{u_{2}} \equiv \frac{\left(p \varphi_{y}-q \varphi_{x}\right)^{3}}{p^{2} t-2 p q s+q^{2} r^{2}}
$$

$$
D_{1} \varphi \equiv \frac{v_{3}^{3}}{v_{1}{ }^{3}} \quad \frac{\left\{\left(s^{2}-r t\right)\left(p \varphi_{y}-q \varphi_{x}\right)+\left(p^{2} t-2 p y s+q^{2} r\right)\left(\varphi_{x}^{2} t-2 \varphi_{x} \varphi_{y} s+\varphi_{y}{ }^{2} r^{2}\right)^{3}\right.}{\left(p^{2} t-2 p q^{s}+q^{2}{ }^{2}\right)^{1}} .
$$

If the operations indicated by these pinameters be performed on $I_{3}, J_{3}, K_{3}$, and $L_{3}$, all essential differential invariants of higher orders can be found by mere differentiation.

We shall make use of the above results in the next section, where we shall conclude our investigations with a discussion of the equivalence of surfaces.

$\$ 5$.

\section{Equivalence of Surfaces.}

14. If a family of surfaces is given by an equation of the form

$$
z=f(x y)
$$

we may always write this equation in the form

$$
\begin{aligned}
z-z_{11}=p_{0}\left(x-x_{0}\right)+q_{0}\left(y-y_{10}\right)+r_{2}^{r_{0}}\left(x-x_{01}\right)^{2}+ & s_{10}\left(x-x_{0}\right)\left(y-y_{0}\right) \\
& +\frac{t_{0}}{2}\left(y-y_{11}\right)^{2}+\ldots
\end{aligned}
$$

where $s_{0}, p_{0} \ldots t_{0} \ldots$ are the values of $a, p \ldots t \ldots$ when we assign to $x$ and $y$ their initial values $x_{v}, y_{v^{\circ}}$.

Now it is clear that the number of surfaces in the family (1) will depend upou the number of arbitrary constants, $z_{0}, p_{0} \ldots t_{0} \ldots$ in equation (2). If the partial differential coefficients $: 2, p, \ldots t \ldots$ are convected by no relation, there will clearly be an unlimited number of surfaces in the family. But if all 
the partial differential coefficients except $n$, are expressed by means of a system of partial differential equations in terms of the remaining $u$ differential coefficients, equation (2) will contain ouly $n$ arbitrary constants ; that is, the family (1) will consist of $\infty^{n}$ surfaces.

Now if we perform all the transformations of the $G_{\mathrm{s}}^{r}$ upon a surface which admits of no transformation of the $G_{s}$, this surface will generate churing the

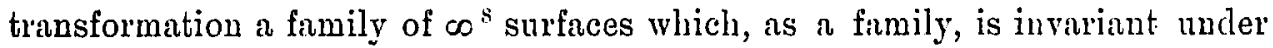
the $G_{\mathrm{s}}$. This family of $\infty^{\mathrm{s}}$ surfiaces

$$
z=F\left(x, y, c_{1} \ldots c_{s}\right)
$$

must be defined by a system of partial differential equatious, which is completely (unbeschrankt) integrable.* There is an infinite number of partial differential equations in this system: but we can suppose them arranged so that beginning with those of the lowest order they proceed to those of higher orders. Also we may assume that from the equations of the pith oriter, all partial differeutial coeflicients of that order cannot be eliminated; and, finally, that the differentiation of one of the equations of the system will always give another equation which bas already been obtained belonging to the system.

The system of equations so arranged will always determine, from a certain point on, all the higher differential coefficients of $z$, with respect to $x$ and $y$, in terms of those of lower order (counting $\approx$ as a differential coefficient of the zero order) ; and as the fumily consists of $\infty$ "surfaces, those of the lower differential coefficients which are connected by no relations caunot exceed eiglut in number.

If the surface upon which we perform the transformations of the $G_{3}$ admits of $n<8$ of these transformations, it will readily be seen that it assumes $\infty^{8-m}$ positions, which will form in invariant family of $\infty^{*-m}$ surfaces. What has been satic above for the fimily of $\infty^{8}$ surfices is equally true for the family of $\infty^{\text {"I-m }}$; that is, the latter family is clefined by an invariant system of completely integrable partial differential equations, which determine all the higher partial differential coefficients of $z$ in terms of $8-m$ of the lower differential coefficients, etc.

If we indicate the partial differential coefficients, up to the forrth order by

$$
z, p, q, \quad \text { r. s, } t, \quad \because, \sigma, \mu, \lambda, \quad R, \&, M, L, N \text {, }
$$

it is clear that when a family of $\omega^{s}$ surfaces is invariant, the system of partial differential equations must contain two partial differential equations of the third order, since there are ten partial differential coefficients up to that order. If these two equations are

$$
Q_{1}^{(3)}(x y=p \ldots \lambda)=0, \quad Q_{2}^{(i)}(x y=p \ldots i)=0
$$


it is clear that the derived equations

$$
\frac{\partial Q_{1}^{(3)}}{\partial x}=0, \quad \frac{\partial Q_{1}^{(3)}}{\partial y}=0, \quad \frac{\partial Q_{2}^{(3)}}{\partial x}=0, \quad \frac{\partial Q_{2}^{(3)}}{\partial y}=0
$$

will determine four of the partial differential coefficients of the fourth order. Hence, to determine a family of exactly $\infty^{8}$ surfaces, we must have the system (3), (4), and one more equation of the fourth order which is not a consequence of $(3),(4)$. This equation has the general form

$$
\left.\Omega_{3}^{(1)}(x y=1) \ldots N\right)=0 \text {; }
$$

and it is clear that all partial differential coefficients of the fifth and higher orders are cletermined in terms of those of lower order's, leaving (say)

connected by no relation.

$$
z, p, q, r, s, t, \|, \sigma
$$

Proceeding in this mauner, we are led to distinguish the following cases:

A. No equation of an order lower than the third oceurs in the invariant system of partial differential equations.

Case $\Gamma$. As we have seen, if two partial differential equations of the third order occur, and one of the fourth (which is not a consequence of the other two) this system of equations is exactly sufficient to determine an invariant family of $\infty^{s}$ surfaces.

Case II. We might have a system of three different partial differential equations of the third order

$$
Q_{1}^{(3)}=0, \quad Q_{2}^{(3)}=0, \quad Q_{3}^{(3)}=0
$$

invariant, and none of lower order: In this case, all differential coefficients of the fourth and higher orders are determined in terms of those of lower orders ; and thus as (say)

$$
. \approx, p, q, r, s, t, "
$$

are connected by no relation, the invariant family consists of $\infty^{7}$ surfaces.

Case ITI. If four differential equations of the third order are invariant, and none of lower orders, the family clearly consists of $\infty^{6}$ surfaces.

These are all the possibilities when no differential equation of an order lower than the third occurs.

B. No differential equation of an order lower than the second occurs.

There is only one invariant partial differential equation of the second orcler, wamely,

The derived equations

$$
F \equiv p^{2} t-2 p q s+q^{2} r^{\prime}=0 \text {. }
$$

$$
F_{x}=0, \quad \dot{F}_{y}=0
$$


WILLIANS. ON THE GEOMELRY OF THE TRANSFORMATION GROUP, ITTC.

determine two of the partial differential coefticients of the third order, and leave two of them arbitrary. MLoreover, the second derived equations

$$
F_{: x x}=0, \quad F_{x y}=0, \quad F_{y y}=0,
$$

determine three of the differential coefficients of the fourth order, and leave two of them arbitrary. In general, we see that two of all the partial differential coefficients of any order, after the second, are arbitrary as firr as $F=0$ and its derived equations are concerued.

Thus when $F=0$ belongs to the invariant system of partial differential equations which define the inviuiant firmily of surfinces, we may assume that up to the fourth order $F=0$ and its derived equations detenmine six of the partial differential coefficients, (say)

$$
t, \mu, \lambda, M, L, N
$$

in terms of those of lower orders.

Case $T$. Hence, if $F=0$ belongs to the invariant system of partial differential equations, it is clear that up to the fourth order, nine of the partial differential coefficients are still arbitrary as far as $F=0$ and its derived equations are concerned. As the fumily cunnot contain more than $\omega^{5}$ surfaces, we must, therefore, have an equation of the fourth order in the system which is not derived from $F=0$.

This equation must, of course, have the form

$$
Q^{(\cdot)}=0 \text {, }
$$

and will determine (say) $s$ in terms of the other differeutial coefficieuts. It is clear that the derived equations of $F=0$ and $\varrho_{1}=0$ determine all the difterential coefficients of the fifth order; so that the invariant system of equations

$$
\begin{aligned}
& F=0, \quad F_{x}=0, \quad F_{y}=0, \quad F_{x, x}=0, \quad F_{x, y}=0, \quad F_{! ! l y}=0, \\
& Q^{(1)}=0, \quad \varrho_{x}^{(1)}=0, \quad Q_{y}^{(1)}=0 \text {, }
\end{aligned}
$$

are exactly enough to cletermine an invariant fumily of $\infty^{8}$ surfices.

Case II. We might have, in connection with $F=0$ aud its derived equations, two partial differential equations of the fourth order invariant, of the forms

$$
\varrho_{1}^{(i)}=0, \quad \varrho^{(+1)}=0 \text {. }
$$

In this case, the only partial differential coeflicients which are arbitrary may clearly be assumed to be (say)

$$
\approx, p, q, r, s, ", \sigma
$$

that is, the family consists of ouly $\infty^{i}$ surfaces.

Case III. In connection with $F=0$ we might have one differential equation of the third order invariant which is not a derived equation of $F^{\prime}=0$. 


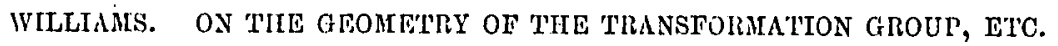

It is clear that in this case this other equation of the third order has the form

$$
\varrho^{(3)}=0 \text {; }
$$

and this equation deternines say $\sigma$. All higher differential coeflicients are now determined in terms of those of lower orders except

$$
z, p, q, r, s, n \text {; }
$$

that is, the fimily consists of $\omega^{6}$ surfaces.

Case $I V$. If two equations of the third order which are not derived from $F=0$, of the general forms

$$
Q_{1}^{(3)}=0, \quad Q_{2}^{(3)}=0
$$

are invariant, the only arbitrary difforential coefficients are

$$
z, p, q, r, s \text {. }
$$

Hence the family consists of os surfices.

These are all the possibilities when no partial differential equation of the first order occurs.

C. Partial differential equations of the first order occur.

No single invariant differential equation of the first orcler exists. The only invariant system containing differential equations of the first order is

$$
p=q=0,
$$

which defines the invariant family of $\infty^{1}$ planes

$$
z=\text { const. }
$$

We may collect our results as follows :

I. If the invariant fitmily consists of $\infty^{*}$ surfaces, it is defined by forms

(a) Two partial differential equations of $I I I .0$. and one $I V .0$., of the

$$
\varrho_{1}\left(I_{3}, J_{3}, K_{3}, I_{3}\right)=0, \quad S_{2}\left(I_{3}, I_{3}, K_{3}, I_{3}\right)=0, \quad \varrho\left(I_{3} \ldots M_{4}\right)=0 .
$$

(b) One partial differential equation of $I I .0$. and one $I V$. 0 . which are respectively

$$
r \equiv p^{2} t-2 p q s+q^{2} r=0 \text {, and } Q\left(I_{3} \ldots M_{4}\right)=0 .
$$

II. If the family consists of $\infty^{i}$ surfaces it is defived by

(a) Thrce partial differential equations $I I I .0$, of the forms

$$
\varrho_{1}\left(\Gamma_{3} \ldots L_{3}\right)=0, \quad \varrho_{2}\left(I_{3} \ldots L_{3}\right)=0, \quad S_{3}\left(I_{3} \ldots L_{3}\right)=0 .
$$

(b) One partial differential equation $I I .0$. and two $I V$. o. of the forms

$$
F=0, \quad \varrho_{1}\left(I_{3} \ldots M_{4}\right)=0, \quad \varrho_{2}\left(I_{3} \ldots M_{4}\right)=0 \text {. }
$$




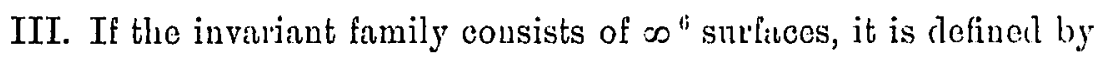

(a) Four partial differential equations $I I J .0$.

$\Omega_{1}\left(I_{3} \ldots I_{3}\right)=0, Q_{2}\left(I_{3} \ldots L_{3}\right)=0, Q_{3}\left(I_{3} \ldots L_{3}\right)=0, Q_{4}\left(I_{3} \ldots L_{3}\right)=0$.

(b) One partial differential equation $I I .0$. and one $I I L .0$.

$$
F=0, \quad \Omega\left(I_{3} \ldots L_{3}\right)=0 \text {. }
$$

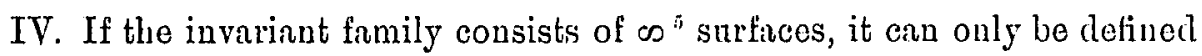
by

(a) One partial differential equation $I I .0$. and two $I \Gamma \Gamma .0$, which have the forms

$$
F=0, \quad \varrho_{1}\left(I_{3} \ldots I_{3}\right)=0, \quad \varrho_{3}\left(I_{3} \ldots I_{33}\right)=0 .
$$

There are no invariant families of $\infty^{4}, \infty \infty^{3}$, or $\infty^{2}$ surfilces; honce no sulface admits of exictly four, five, or six independent infinitesimal transformations of the $G_{\mathrm{s}}$. There is then only one other case.

V. The invariant family consists of the $s^{1}$ planes $z=$ const. defined by

$$
p=\eta=0 \text {. }
$$

From the above considerations we reach tho following important conclnsion :

If two surficces are equivalent by means of the transformations of the $G_{\text {s }}$, their equations must both satisfy the partical differential eyutions enumerated in some one of the above cuses I . . V.

Wo may note in this connection that when

$$
p^{2} t-2 p q s+q^{2} r=0
$$

the invariant, $I_{3}$, becomes elusive, as do also $A_{1} \varphi$ and $D_{1} \varphi$. But

$$
\frac{D_{1} \varphi}{J_{1} \varphi}
$$

is a differential parameter which is not elnsive; and this applied to $J_{3}, K_{3}$, or $I_{3}$ (some of them are certainly not elusive) will give differential invariants of tho fourtl and higher orders. In this way we can find all differential invalriants of the third aud fourth orders which are not zero on account of (5); and arbitrary functions of these differential invariants will bo the invariant equations required in the system of partial differential equations defining the inviriant fimilies of surfinces.

Wo know also that (5) represents on ruled surfuces; and this unlimited assemblage of rnled surlites is divided into fiumilies of equivilent surfices by means of the other differential equations of the particular invariant system. 